\title{
Ground Nonmonotonic Modal Logics
}

\author{
FRANCESCO M. DONINI, DANIELE NARDI and RICCARDO ROSATI, \\ Dipartimento di Informatica e Sistemistica, Università di Roma "La \\ Sapienza”, Via Salaria 113, 00198 Roma, Italy
}

\begin{abstract}
In this paper we address ground logics, a family of nonmonotonic modal logics, and their usage in knowledge representation. In such a setting non-modal sentences are used to represent the knowledge of an agent about the world, while an epistemic operator provides the agent with autoepistemic or introspective knowledge. Ground logics are based on the idea of characterizing the knowledge of the agent by allowing it to make nonmonotonic assumptions only with respect to the knowledge about the world, i.e. expressed by nonmodal formulae. They are characterized by a fix-point equation which determines the set of formulae derivable from the agent's initial knowledge and which can be applied to different normal modal systems to obtain a variety of nonmonotonic modal logics. In the paper we address the semantical, computational and epistemological properties of ground logics. We provide a semantic characterization of ground logics by defining a preference relation on possible-world models based on the minimization of the knowledge expressed by nonmodal formulae. We analyze the computational complexity of reasoning in ground logics, providing both a lower bound through a reduction from quantified boolean formulae and an upper bound through an algorithm for computing logical entailment. We discuss the representational features of ground logics, in particular defaults, and provide a thorough comparison with McDermott and Doyle's logics.
\end{abstract}

Keywords: Knowledge representation, nonmonotonic reasoning, autoepistemic logics, computational complexity.

\section{Introduction}

The interplay between nonmonotonic reasoning and reasoning about knowledge and belief has been recognized from the beginning of the research on commonsense reasoning. Since then, modal epistemic logics have been studied with the aim of characterizing the reasoning of an agent who is capable to perform introspective reasoning by making assumptions on its own knowledge. Non-modal sentences represent the knowledge of the agent about the world, while an epistemic operator provides the agent with auto-epistemic or introspective knowledge.

The first formalizations of nonmonotonic reasoning based on the use of a modal operator have been proposed in $[22,21,24]$. The knowledge of an agent is characterized in terms of a fix-point equation which determines the set of formulae derivable from the agent's initial knowledge; such equation formalizes an agent having full introspective capabilities about its own lack of knowledge. Ground logics [29, 33, 13, 36, 14] have later been defined by restricting the introspection of the agent to nonmodal sentences. The term ground logic refers to the idea of enabling the agent only to make assumptions that are grounded in the world's knowledge. The notion of groundedness has been introduced in [14] and has a rather intuitive motivation: in fact, it corresponds to discarding any reasoning based on epistemic assumptions, which, for example, would enable the agent to conclude that something is true in the world, by assuming to know it.

A different approach for defining nonmonotonic modal logics was taken in [11, 32, 15], where the knowledge of the agent is characterized on a semantic basis, by means of a preference criterion among the models of the agent's initial knowledge. Many of such criteria follow the idea of selecting those models in which knowledge is minimal. 
Recently, there have been a number of attempts to reconcile fix-point and semantic characterizations of modal nonmonotonic logics. In particular, Schwarz [30] proposed a semantics for McDermott and Doyle's logics. However, the notion of minimal knowledge underlying the above cited works is stronger than the one used to characterize McDermott and Doyle's logics. In particular, for the modal system S5, McDermott and Doyle's equation does not provide a nonmonotonic logic, while S5 models of minimal knowledge have a natural interpretation as maximal sets of possible worlds.

The goal of our work ${ }^{1}$ is to study the family of ground logics, from the semantical, computational and epistemological viewpoint. With respect to the first issue, we present an appropriate semantic characterization for ground logics, that has been advocated in [31]. In particular, our proposal is an instance of the preference semantics introduced by Shoham [32], where the preference criterion is given by a partial ordering over possible-world models and generalizes the idea of minimal knowledge as proposed in $[11,32,15]$. We show the correspondence between such semantic characterization and the fix-point definition of ground logics for a subclass of normal modal logics, called cluster-decomposable logics, which includes the most studied cases in nonmonotonic modal logics.

As for the computational properties of ground logics, we show that reasoning in ground logics is $\Pi_{3}^{p}$-hard. Comparing this result with computational complexity analyses of McDermott and Doyle's logics $([8,26,20])$, it turns out that ground logics are computationally harder than the corresponding McDermott and Doyle's logics. We prove that $\Pi_{3}^{p}$ is also an upper bound for the major ground nonmonotonic logics, namely S5, S4F, SW5 (the same result for KD45 was shown in [5]), by providing an algorithm for computing logical entailment. In particular, we provide the computational characterization of the logic of minimal knowledge initially proposed in [11].

Based on the above characterization we discuss some properties of ground logics, specifically addressing their semantics, the complexity of reasoning, the treatment of defaults and the use of definitions - which has been considered a problematic aspect of these logics [31]. This discussion and a comparison with McDermott and Doyle's logics show that the idea of minimizing knowledge in terms of the world knowledge of the agent gives rise to several interesting features from the viewpoint of knowledge representation. In particular, the restriction to grounded assumptions can be regarded as a a refinement of the introspection capabilities provided by McDermott and Doyle's logics. The non-triviality of this additional selection is confirmed both by the semantical and by the computational analysis: on the one hand we have a greater variety of ground nonmonotonic logics, on the other hand reasoning is harder. However, we identify some special cases of practical interest, where the complexity of reasoning is lower than in the general case. The differences between ground logics and McDermott and Doyle's logics are further highlighted by their ability to represent defaults. While in the general case they have a similar behaviour they are somewhat complementary for special forms of defaults: Ground logics naturally capture justification-free defaults, while McDermott and Doyle's logics allow for a simple formalization of prerequisite-free defaults.

The paper is organized as follows. In the next section we report on previous work on nonmonotonic modal logics, including a fix-point definition of ground logics. We then present our semantic characterization in terms of a preference criterion on possible-world structures. In the subsequent section we address the computational aspects of reasoning in such logics. We finally discuss some interesting properties of ground logics and compare them with McDermott and Doyle's logics.

\footnotetext{
${ }^{1}$ This work is an abridged and extended version of $[25,4]$.
} 


\section{Nonmonotonic modal logics}

In this section we recall the relevant background on nonmonotonic modal logics and introduce ground logics.

\subsection{Notation}

We use $\mathcal{L}$ to denote a fixed propositional language with propositional connectives $\vee, \wedge, \neg, \supset$, and whose generic atoms are denoted as $p, q, x, y, z$ (possibly with subscripts). Formulae over $\mathcal{L}$ will be often called objective, because they do not contain occurrences of the modal operator. A propositional valuation for $\mathcal{L}$ is a function that assigns to every atom of $\mathcal{L}$ one of the truth values true, false. Propositional valuations are denoted with symbols $u, v$, and can be extended to propositional formulae in the usual way.

We denote with $\mathcal{L}_{K}$ the modal extension of $\mathcal{L}$ with the only modality $K$ (for knowledge). Generic formulae over $\mathcal{L}_{K}$ will be denoted as $\varphi, \psi$. A propositional valuation over $\mathcal{L}_{K}$ is a function that assigns a truth value to every atom of $\mathcal{L}$, and to any formula of the form $K \varphi$. Also these valuations are denoted with symbols $u, v$, and are extended to modal formulae as follows: first substitute the modal subformulae having the outermost modal operators (i.e., the subformulae of the form $K \varphi$ not appearing in the scope of any $K$ ) with their truth values, then substitute the remaining propositional atoms with their truth values, and then compose such values with the usual rules for propositional connectives.

For example, let $\varphi$ be the formula $p \vee \neg K(z \wedge \neg K y)$, and let $v$ assign the truth values $v(p)=$ false, $v(K(z \wedge \neg K y))=$ true. Then $v(\varphi)=$ false. Observe that values assigned to $z, y$ and $K y$ do not matter for propositional valuations of this formula.

Sometimes we use partial valuations, i.e., valuations assigning a truth value only to some atoms and modal formulae. Given a formula $\varphi$ and a partial valuation $v$, we denote with $\varphi_{v}$ the formula obtained by substituting (as described above) in $\varphi$ those atoms and subformulae defined in $v$ with their truth values, and simplifying when possible. Obviously, when $v$ is a (total) propositional valuation, $\varphi_{v}$ is just a truth value.

A propositional valuation $v$ over $\mathcal{L}_{K}$ satisfies a formula $\varphi$ if $v(\varphi)=$ true. We say that a formula $\varphi$ over $\mathcal{L}_{K}$ is propositionally consistent if there is a propositional valuation $v$ over $\mathcal{L}_{K}$ such that $v$ satisfies $\varphi$. Observe that there are modal formulae, such as $p \wedge \neg K p$, which are propositionally consistent, although they are not consistent in any normal modal logic. When we want to restrict a propositional valuation $v$ over $\mathcal{L}_{K}$ to propositional atoms only, we write $v_{\mid \mathcal{L}}$.

We extend the above definitions to a set of formulae $T$ over $\mathcal{L}_{K}$ in the usual way: $v(T)$ is the logical conjunction of the values assigned by $v$ to each formula in $T$. Given a set of formulae $T$, we denote with $C n(T)$ the set of all propositional consequences of $T$, i.e., formulae which are satisfied by every propositional valuation satisfying $T$.

A Kripke model $\mathcal{M}$ is defined as usual by a triple $\langle W, R, V\rangle$, where $W$ is a set (whose elements are called worlds), $R$ is a binary relation on $W$ (called the accessibility relation on $\mathcal{M}$ ), and $V$ is a function assigning a propositional valuation to each world $w \in W$. When $R$ is $W \times W$ (i.e. $\mathcal{M}$ is a universal model) we simply write $\langle W, V\rangle$.

We define the satisfiability relation between a modal formula $\varphi$ and a world $w$ belonging to the worlds of a Kripke model $\mathcal{M}$ (denoted by $\langle\mathcal{M}, w\rangle \models \varphi$ ) in the following way:

1. if $\varphi$ is a propositional symbol, then $\langle\mathcal{M}, w\rangle \models \varphi$ iff $V(w)(\varphi)=$ true; 
2. if $\varphi=\neg \psi$, then $\langle\mathcal{M}, w\rangle \models \varphi$ iff $\langle\mathcal{M}, w\rangle \models \psi$ is not true;

3. if $\varphi=\psi_{1} \vee \psi_{2}$, then $\langle\mathcal{M}, w\rangle \models \varphi$ iff $\langle\mathcal{M}, w\rangle \models \psi_{1}$ or $\langle\mathcal{M}, w\rangle \models \psi_{1}$;

4. if $\varphi=\psi_{1} \wedge \psi_{2}$, then $\langle\mathcal{M}, w\rangle \models \varphi$ iff $\langle\mathcal{M}, w\rangle \models \psi_{1}$ and $\langle\mathcal{M}, w\rangle \models \psi_{1}$;

5. if $\varphi=K \psi$, then $\langle\mathcal{M}, w\rangle \models \varphi$ iff for every $w^{\prime}$ such that $\left(w, w^{\prime}\right) \in R,\left\langle\mathcal{M}, w^{\prime}\right\rangle \models \psi$.

We say that a Kripke model $\mathcal{M}$ satisfies $\varphi$ (written $\mathcal{M}=\varphi$ ) iff for all $w \in \mathcal{M},\langle\mathcal{M}, w\rangle \models \varphi$.

We denote with $T h(\mathcal{M})$ the set of formulae of $\mathcal{L}_{K}$ that are satisfied in $\mathcal{M}$, i.e., $T h(\mathcal{M})=$ $\left\{\varphi \in \mathcal{L}_{K} \mid \mathcal{M} \models \varphi\right\}$.

Given a modal logic $\mathcal{S}$, we denote with $C n_{\mathcal{S}}$ the consequence operator in (classical) modal logic $\mathcal{S}$. Given two modal logics $\mathcal{S}_{1}$ and $\mathcal{S}_{2}$, by $\mathcal{S}_{1} \subseteq \mathcal{S}_{2}$ we mean that all axioms of $\operatorname{logic} \mathcal{S}_{1}$ are also axioms (or theorems) in logic $\mathcal{S}_{2}$. E.g., it is known that $\mathrm{K} \subseteq \mathrm{KD} 45 \subseteq \mathrm{S} 5$.

In the following, we call S5-model a Kripke model whose accessibility relation is universal, i.e. each world is connected to all worlds of the model. The class of universal models characterizes the logic S5 (see for example [20, Theorem 7.52]).

Throughout the paper, the set of formulae $I \subseteq \mathcal{L}_{K}$ stands for the initial knowledge of the agent.

\subsection{McDermott and Doyle's logics}

We start by recalling McDermott and Doyle's equation which applies to the consequence operator of a monotonic modal logic $[22,21]$. The equation is a general scheme for defining expansions, namely possible sets of sentences representing the knowledge of an agent reasoning introspectively from an initial body of knowledge.

Given any modal logic $\mathcal{S}$, a consistent set of formulae $T$ is an $\mathcal{S}_{M D D^{-}}$expansion for a set of initial knowledge $I \subseteq \mathcal{L}_{K}$ if $T$ satisfies the following equation:

$$
T=C n_{\mathcal{S}}\left(I \cup\left\{\neg K \varphi \mid \varphi \in \mathcal{L}_{K} \backslash T\right\}\right),
$$

The resulting consequence operator $\models_{\mathcal{S}_{M D D}}$ is defined as the intersection of all $\mathcal{S}_{M D D^{-}}$ expansions for $I$. Such operator is in general nonmonotonic: thus for every modal logic $\mathcal{S}$, the (nonmonotonic) modal logic $\mathcal{S}_{M D D}$ is obtained by means of equation (1).

The McDermott and Doyle's family of nonmonotonic modal logics has been extensively studied [24, 19, 18, 20]. McDermott [21] analyzed the case of $\mathcal{S}=$ S5 and found out that the resulting logic $\mathrm{S} 5_{M D D}$ is monotonic, in the sense that the intersection of all $\mathrm{S} 5_{M D D^{-}}$ expansions of a theory $I$ is exactly the set of consequences of $I$ in monotonic S5. Schwarz [28] proved the equivalence of Moore's autoepistemic logic [24] with logic KD45 $5_{M D D}$. He also defined in [30] a preference semantics for McDermott and Doyle's family, thus giving a true possible-world semantic characterization of this class of modal nonmonotonic formalisms.

This result allows one to study properties of logics $\mathcal{S}_{M D D}$ by reasoning on possible-world structures, which is often easier than analyzing infinite sets of modal formulae. From this semantic viewpoint it is easy to show that $S 5_{M D D}$ is not the only degenerate (i.e. monotonic) case for logics $\mathcal{S}_{M D D}$. More specifically, there is a whole class of logics in the McDermott and Doyle's family degenerating to monotonic S5, precisely the logics characterized by a class of Kripke models whose accessibility relation is symmetric, i.e. all logics $\mathcal{S}_{M D D}$ such that every instance of the modal axiom schema $\mathrm{B}(\neg K \neg K \varphi \supset \varphi)$ is valid in $\mathcal{S}$. 


\subsection{Ground logics}

Let us now turn the attention to the nonmonotonic modal logics that have been proposed on the basis of semantic considerations and, more precisely, on the intuition that in the models the knowledge attributed to the agent should be minimal. This principle was introduced in [11] and it was enforced by minimizing the set of objective (i.e. nonmodal) sentences known by the agent. This notion of minimal knowledge can be stated in terms of a property on the models of a logical theory $I$ as follows:

Definition 2.1 (Minimal Knowledge) A model $\mathcal{M}$ is a model of minimal knowledge for $I \subseteq \mathcal{L}_{K}$ in the logic $\mathcal{S}$, if $\mathcal{M}$ is a model for $I$ in $\mathcal{S}$ and for every model $\mathcal{M}^{\prime}$ of $I$ in $\mathcal{S}$, $T h\left(\mathcal{M}^{\prime}\right) \cap \mathcal{L} \not \subset T h(\mathcal{M}) \cap \mathcal{L}$.

We say that a logic $\mathcal{S}$ is a logic of minimal knowledge if for every theory $I \subseteq \mathcal{L}_{K}$, every model for $I$ in $\mathcal{S}$ is a model of minimal knowledge for $I$ in $\mathcal{S}$.

The first modal logic of minimal knowledge was introduced by Halpern and Moses in [11], and is based on a simple and "natural" preference semantics on modal logic S5 [32, 15], which realizes the intuitive principle of minimization of the knowledge of the agent modeled. Such a logic, initially proposed for modeling knowledge and ignorance of processes in a distributed computer system, constitutes the basis of several nonmonotonic modal formalisms proposed in the literature [15, 17, 6, 23].

In [15] a possible-world semantics for this logic is given as follows. Let the possible worlds be the propositional valuations over $\mathcal{L}$. Each model $\mathcal{M}=\langle W, R, V\rangle$ is such that $R=W \times W$ and $V(v)=v$, i.e. $V$ is the identity function. That is, $\mathcal{M}$ is fully characterized by its set of worlds $W$. This amounts to considering universal (S5) models, i.e. connected structures whose accessibility relation is reflexive, symmetric and transitive. A sentence $K \varphi$ is true in a world $w$ belonging to $\mathcal{M}$ if $\varphi$ is true in all worlds $w^{\prime}$ belonging to $\mathcal{M}$. However, not every universal S5-structure that satisfies the initial assumptions $I$ of the agent is taken into consideration: the interesting models are the maximal ones, namely those which do not have any proper superset satisfying $I$. In other words, an S5-model is of minimal knowledge if it satisfies $I$ and cannot be extended by adding a new possible world (i.e. another propositional valuation of $\mathcal{L}$ ). Therefore, minimization of knowledge is obtained by maximizing the set of possible worlds, sometimes explained as maximizing ignorance. The nonmonotonic character of this construction becomes evident when looking at the case in which $I$ is the empty set. In this case one can conclude $\neg K p$ for every atom $p$, but this conclusion does not hold anymore when $p$ is added to $I$.

This idea has been further developed by Lifschitz in [15] and by Lin and Shoham in [17], where a bimodal logic that combines minimization of knowledge with justified assumptions is proposed However, the more recent version [16] of Lifschitz's work [15] contains a technical difference which makes the resulting logic a logic of "minimal belief", that is no longer captured by the above definition (see also [1] where such a logic is rephrased using a single modal operator). In [31] the minimization of knowledge is formulated in terms of a preference criterion on Kripke models which differs from the superset criterion presented above: notably, the class of models taken into consideration is the class of models characterizing modal logic S4F. However, this way of minimizing knowledge does not correspond to the minimization of objective sentences, so this logic is not a logic of minimal knowledge in the sense of Definition 2.1.

The idea of minimal knowledge can be naturally captured also by a fix-point equation in the McDermott and Doyle's style, by bounding introspection in the right-hand side of 
Equation (1) to objective formulae only. Given a normal modal logic $\mathcal{S}$, a consistent set of formulae $\mathrm{T}$ is a ground $\mathcal{S}$-expansion (from now on, $\mathcal{S}_{G}$-expansion for short) for a set $I \subseteq \mathcal{L}_{K}$ if $T$ satisfies the following equation:

$$
T=C n_{\mathcal{S}}(I \cup\{\neg K \varphi \mid \varphi \in \mathcal{L} \backslash T\}) .
$$

This equation, which was first used by Konolige in the case $\mathcal{S}=$ KD45 [14], defines a family of logics $\mathcal{S}_{G}$ called ground nonmonotonic modal logics [29, 33, 13] (ground logics for short).

Also in this case, the agent's initial knowledge $I$ entails a formula $\varphi$ (written $I \models \mathcal{S}_{G} \varphi$ ) if $\varphi$ belongs to all the $\mathcal{S}_{G}$-expansions for $I$.

Every $T$ satisfying fix-point Equation (2) satisfies also Equation (1), i.e., every $\mathcal{S}_{G^{-}}$ expansion is also an $\mathcal{S}_{M D D}$-expansion [20, Theorem 11.30]-but not vice versa, as shown by examples below. Therefore ground logics are more selective than McDermott and Doyle's logics, since they admit fewer expansions.

In fact, ground logics are logics of minimal knowledge in the sense of Definition 2.1, as stated by the following proposition, which directly follows from a property of minimality of $\mathcal{S}_{G}$-expansions [20, Theorem 11.36].

Proposition 2.2 Let $T \subseteq \mathcal{L}_{K}$ be a theory and $\mathcal{M}$ be a model such that $T h(\mathcal{M})=T$. If $T$ is an $\mathcal{S}_{G}$-expansion for $I \subseteq \mathcal{L}_{K}$, then $\mathcal{M}$ is a model of minimal knowledge for $I$.

Therefore, ground logics are logics of minimal knowledge, while McDermott and Doyle's logics are not, as shown by the following example, where the agent is able to conclude that it does not know a piece of world knowledge, when it lacks information about it.

Example 2.3 Let $I$ be empty, i.e. $I=\emptyset$. Since $\mathrm{S} 5_{M D D}$ degenerates to $\mathrm{S} 5$, every $\mathrm{S} 5$-model is a model for $I$ in $\mathrm{S} 5_{M D D}$, whereas only the (maximal) S5-model containing all possible worlds is a (minimal-knowledge) model for $I$ in $\mathrm{S} 5_{G}$. Therefore, for every $\varphi$ which is not an S5 theorem, we have $I \not \|_{\mathrm{S} 5_{M D D}} \neg K \varphi$ while $I=_{\mathrm{S}_{G}} \neg K \varphi$. This captures the intuition that, without any premises, the agent can prove that it does not know any formula, but S5 theorems.

The different behaviour of ground logics and McDermott and Doyle's logics is further illustrated by the following examples, that are related to the notion of groundedness.

Example 2.4 Let $I=\{K p \supset p\}$. For every logic $\mathcal{S}$ containing the modal axiom schema 5, we show that there are at least two $S_{M D D}$-expansions for $I$, namely, $T_{1}=\{p, K p, \ldots\}$ and $T_{2}=\{\neg K p, \ldots\}$. In fact, if $p \in T_{1}$ then:

(a) $K p \in T_{1}$ (by necessitation)

(b) $\neg K p \notin T_{1}$ (by consistency of $T_{1}$ )

(c) $\neg K \neg K p \in C n_{\mathcal{S}}\left(I \cup\left\{\neg K \varphi \mid \varphi \in \mathcal{L}_{K} \backslash T_{1}\right\}\right)$

(d) $K p \in C n_{\mathcal{S}}\left(I \cup\left\{\neg K \varphi \mid \varphi \in \mathcal{L}_{K} \backslash T_{1}\right\}\right.$ ) (from axiom 5, by contraposition)

(e) $p \in C n_{\mathcal{S}}\left(I \cup\left\{\neg K \varphi \mid \varphi \in \mathcal{L}_{K} \backslash T\right\}\right.$ ) (from $I$ ),

hence $T_{1}$ is a fix-point of (1). To verify that $T_{2}$ is a fixpoint, it is sufficient to observe that if $p \notin T_{2}$ then $\neg K p \in T_{2}$, then premise $K p \supset p$ cannot be used to derive $p$. From the fact that $T_{1}$ is an $\mathcal{S}_{M D D}$-expansion, $I \not \forall_{\mathcal{S}_{M D D}} \neg K p$ when $\mathcal{S}$ contains schema 5 .

On the other hand, for every ground logic $\mathcal{S}_{G}, T_{1}$ is not an $\mathcal{S}_{G}$-expansion for $I$, because step (c) is not valid for Equation (2): $\neg K p \notin \mathcal{L} \backslash T$. Thus, $T_{2}$ is the only $\mathcal{S}_{G}$-expansion and 
therefore, there is no $\mathcal{S}_{G}$-expansion in which $p$ is true, hence $I \models \mathcal{S}_{G} \neg K p$. Observe that this agrees with the intuition that $T_{2}$ is a minimal-knowledge expansion (it contains no objective theorems but tautologies).

As pointed out in [14], in this case the reasoning that leads the agent to derive $p$ in one $\mathcal{S}_{M D D}$-expansion is ungrounded, since by assuming to know $p$ the agent derives $p$ itself. Also in the next example, the agent, by making assumptions on its epistemic knowledge about $p$, achieves $p$ as a conclusion and thus fails to derive $\neg K p$.

Example 2.5 Let $I=\{\neg K \neg K p \supset p\}$. For every logic $\mathcal{S}_{M D D}$ we have that there exists an $\mathcal{S}_{M D D}$-expansion for $I$ containing $p$, i.e. $I \not \forall_{\mathcal{S}_{M D D}} \neg K p$. On the other hand, for every ground logic $\mathcal{S}_{G}$ there is no $\mathcal{S}_{G}$-expansion for $I$ containing $p$, hence $I \models{ }_{\mathcal{S}_{G}} \neg K p$.

The above examples point out some differences between ground logics and McDermott and Doyle's logics, which motivate the study of ground logics. A more detailed comparison between the two is deferred after addressing the preference semantics (in the next section) and the computational complexity of reasoning in ground logics (in Section 4).

\section{Minimal model semantics}

We now present the semantic characterization for a relevant subset of ground nonmonotonic modal logics, by providing a preference relation on Kripke models. The path we follow is similar to the one used in $[30,20]$, to provide a semantic characterization for the McDermott and Doyle's family of logics. We first recall some definitions and previous results that are used in the rest of the section. Then, we introduce a notion of ground-intended model, that constitutes a first step towards the semantic characterization of theories formulated through a fix-point equation. Subsequently, we focus on the preference relation, starting from the intuition that the syntactic notion of minimization of objective sentences can be formulated in terms of a partial ordering relation on Kripke models. We strengthen the preference relation defined in [30], by weakening the preconditions for the comparison of Kripke models and thus allowing more models to be compared. The preference relation is then used to characterize ground-minimal models. The final step shows the correspondence between ground-intended and ground-minimal models and therefore between the theory defined by the fix-point equation and the preferred possible-world models.

\subsection{Preliminary definitions and previous results}

In order to make the paper self-contained we recall some basic definitions and theorems which are referred to in the following (see [20] for further details).

We start with the definition of stable theory and of its canonical model.

Definition 3.1 A theory $T \subseteq \mathcal{L}_{K}$ is stable if

1. $T$ is closed under propositional consequence;

2. for every $\varphi \in \mathcal{L}_{K}$, if $\varphi \in T$ then $K \varphi \in T$;

3. for every $\varphi \in \mathcal{L}_{K}$, if $\varphi \notin T$ then $\neg K \varphi \in T$. 
Let $S \subseteq \mathcal{L}$. We denote $S T(S)$ the (unique) stable theory $T$ such that $T \cap \mathcal{L}=\operatorname{Cn}(S)$.

Definition 3.2 The canonical model for a stable theory $\mathrm{T}$ is a Kripke model $\mathcal{M}=\langle W, R, V\rangle$ such that $W$ consists of all propositional valuations in which all formulae from $T \cap \mathcal{L}$ are true, $R$ is the universal relation on $W$ (hence $\mathcal{M}$ is an S5-model) and $V(w)=w$ for every $w \in W$.

We recall the following properties of stable theories.

Proposition 3.3 $[20,8.10]$ Let $\mathcal{M}$ be an S5-model. Then $T h(\mathcal{M})$ is a stable theory.

Stable models are strictly related both to MDD-expansions and to ground expansions.

Proposition 3.4 If $T \subseteq \mathcal{L}_{K}$ is an $\mathcal{S}_{M D D}$-expansion for some $I \subseteq \mathcal{L}_{K}$, then $T$ is a stable theory.

Proposition 3.5 Let $\mathcal{S}$ be a modal logic such that $\mathrm{K} \subseteq \mathcal{S} \subseteq \mathrm{S} 5$ and let $I \subseteq \mathcal{L}_{K}$. A theory $T \subseteq \mathcal{L}_{K}$ is an $\mathcal{S}_{G}$-expansion for $I$ iff $T$ is stable, consistent, $I \subseteq T$ and $T \cap \mathcal{L} \subseteq C n_{\mathcal{S}}(I \cup$ $\{\neg K \varphi \mid \varphi \in \mathcal{L} \backslash T\})$.

Proposition 3.6 Given two modal logics $\mathcal{S}^{\prime}$ and $S^{\prime \prime}$ such that $\mathrm{K} \subseteq \mathcal{S}^{\prime} \subseteq \mathcal{S}^{\prime \prime} \subseteq \mathrm{S} 5$, and given a theory $I \subseteq \mathcal{L}_{K}$, every $\mathcal{S}_{G}^{\prime}$-expansion for $I$ is also an $\mathcal{S}_{G}^{\prime \prime}$-expansion for $I$.

We also need the following property, which relates stable theories with Kripke models.

Proposition 3.7 [20, 8.16] Let $T \subseteq \mathcal{L}_{K}$ be a stable theory. Let $\mathcal{M}$ be a Kripke model such that $\mathcal{M} \models(T \cap \mathcal{L}) \cup\{\neg K \varphi \mid \varphi \in \mathcal{L} \backslash T\}$. Then, for every $\varphi \in \mathcal{L}_{K}, \varphi \in T$ iff $\mathcal{M} \models \varphi$, namely $T=T h(\mathcal{M})$.

The following definition characterizes the class of modal logics satisfying the terminal cluster property. The importance of such logics, as stated by subsequent Proposition 3.9, is that, for such logics, ground expansions coincide with those theories that are both $\mathrm{S}_{G^{-}}$ expansions and $\mathcal{S}_{M D D}$-expansions.

Definition 3.8 A logic $\mathcal{S}$ characterized by a class $\mathcal{C}$ of models satisfies the terminal cluster property if for every $\mathcal{M}=\langle W, R, V\rangle \in \mathcal{C}$ and for every world $w \in W$ there is a terminal cluster for $w$, i.e. a maximal subset $Y$ of $W$ such that $Y \times Y \subseteq R$ and:

1. for every $w^{\prime} \in Y,\left(w, w^{\prime}\right) \in R$;

2. for every $w^{\prime} \in Y$ and every $w^{\prime \prime} \in W \backslash Y,\left(w^{\prime}, w^{\prime \prime}\right) \notin R$.

Proposition 3.9 Given a modal logic $\mathcal{S}$ such that $\mathrm{K} \subseteq \mathcal{S} \subseteq \mathrm{S} 5$, if $\mathcal{S}$ satisfies the terminal cluster property then a theory $T$ is an $\mathcal{S}_{G}$-expansion for a theory $I \subseteq \mathcal{L}_{K}$ iff $T$ is both an $\mathcal{S}_{M D D}$-expansion for $I$ and an $\mathrm{S}_{G}$-expansion for $I$.

Notice that modal logics KD45, S4F and SW5 satisfy the terminal cluster property.

Finally, to define a possible-world semantics based on a preference relation we need the ability to compare Kripke models. For this purpose we introduce an operation between Kripke models, called concatenation, which returns a Kripke model. 
Definition 3.10 Given Kripke models $\mathcal{M}_{1}=\left\langle W_{1}, R_{1}, V_{1}\right\rangle, \mathcal{M}_{2}=\left\langle W_{2}, R_{2}, V_{2}\right\rangle$, such that $W_{1} \cap W_{2}=\emptyset$, the concatenation of $\mathcal{M}_{1}$ and $\mathcal{M}_{2}$, written as $\mathcal{M}_{1} \odot \mathcal{M}_{2}$, is the Kripke model $\mathcal{M}=\langle W, R, V\rangle$ such that $W=W_{1} \cup W_{2}, V=V_{1} \cup V_{2}$ and $R=R_{1} \cup\left(W_{1} \times W_{2}\right) \cup R_{2}$.

In addition, we identify the Kripke models which can be decomposed in a particular form, namely in such a way that every model can be defined as the composition of two models, the second one being a universal S5-model.

Definition 3.11 A class $\mathcal{C}$ of Kripke models is cluster-decomposable if every model in $\mathcal{C}$ is of the form $\mathcal{M}_{1} \odot \mathcal{M}_{2}$, where $\mathcal{M}_{2}$ is a universal Kripke model, and for every such model $\mathcal{M}_{1} \odot \mathcal{M}_{2}$ and every universal model $\mathcal{M}_{2}^{\prime}$ whose set of worlds is disjoint from that of $\mathcal{M}_{1}$, the model $\mathcal{M}_{1} \odot \mathcal{M}_{2}^{\prime}$ is in $\mathcal{C}$.

It is easy to see that most of the modal logics studied in the nonmonotonic setting, in particular the logics S5, KD45, S4F and SW5, are all characterized by a cluster-decomposable class of Kripke models.

\subsection{Ground-intended models}

Our first goal is to establish a correspondence between the solutions of the fix-point definition of ground logics and their intended models. Therefore, we introduce the notion of groundintended model, which follows from the properties a reasoning agent should satisfy, namely the initial knowledge $I$ is satisfied and the introspection capability of the agent is restricted to objective formulae only.

Definition 3.12 Given a normal modal logic $\mathcal{S} \subseteq$ S5 characterized by the class $\mathcal{C}$ of Kripke models and a theory $I \subseteq \mathcal{L}_{K}$, a model $\mathcal{M} \in \mathcal{C}$ is ground $\mathcal{C}$-intended for $I$ iff:

1. $\mathcal{M} \models I$;

2. for every model $\mathcal{N} \in \mathcal{C}$, if $\mathcal{N} \models I \cup\{\neg K \varphi \mid \varphi \in \mathcal{L} \backslash T h(\mathcal{M})\}$, then $T h(\mathcal{M})=$ $T h(\mathcal{N})$.

Then, we show that the notion of ground $\mathcal{C}$-intended model exactly corresponds to that of $\mathcal{S}_{G}$-expansion, if $\mathcal{C}$ is the class of Kripke models characterizing modal logic $\mathcal{S}$.

Theorem 3.13 Given a modal logic $\mathcal{S}$ such that $\mathrm{K} \subseteq \mathcal{S} \subseteq \mathrm{S} 5$, let $\mathcal{C}$ be the class of Kripke models characterizing $\mathcal{S}$. An $\mathrm{S} 5$-model $\mathcal{M}$ is a ground $\mathcal{C}$-intended model for $I \subseteq \mathcal{L}_{K}$ iff $T h(\mathcal{M})$ is an $\mathcal{S}_{G}$-expansion for $I$.

Proof We abbreviate $T h(\mathcal{M})$ as $T$ in the proof.

Only-if part. Assume $\mathcal{M}$ is ground $\mathcal{C}$-intended for $I$. Since $\mathcal{M}$ is an S5-model, by Proposition 3.3, $T$ is stable; hence $T \supseteq\{\neg K \varphi \mid \varphi \in \mathcal{L} \backslash T\}$. Besides, from $\mathcal{M} \models I$ it follows that $I \subseteq T$. Since $\mathcal{S} \subseteq \mathrm{S} 5$, it follows that

$$
T \supseteq C n_{\mathcal{S}}(I \cup\{\neg K \varphi \mid \varphi \in \mathcal{L} \backslash T\})
$$

Since $\mathcal{M}$ is ground $\mathcal{C}$-intended, every model of $I \cup\{\neg K \varphi \mid \varphi \in \mathcal{L} \backslash T\}$ in $\mathcal{C}$ is a model of $T$, therefore

$$
T \subseteq C n_{\mathcal{S}}(I \cup\{\neg K \varphi \mid \varphi \in \mathcal{L} \backslash T\})
$$


Hence, $T$ is an $\mathcal{S}_{G}$-expansion for $I$.

If part. Assume that $T=T h(\mathcal{M})$ is an $\mathcal{S}_{G}$-expansion for $I$. Then, $T=C n_{\mathcal{S}}(I \cup\{\neg K \varphi \mid$ $\varphi \in \mathcal{L} \backslash T\}$ ). Consider a model $\mathcal{N} \in \mathcal{C}$ such that $\mathcal{N} \models I \cup\{\neg K \varphi \mid \varphi \in \mathcal{L} \backslash T\}$; it follows that $T \subseteq T h(\mathcal{N})$. And if $\varphi \in \mathcal{L} \backslash T$, then $\mathcal{N} \models \neg K \varphi$, which implies that $\mathcal{N} \not \models \varphi$. Therefore $\mathcal{L} \backslash T \subseteq\{\varphi \in \mathcal{L} \mid \mathcal{N} \forall \forall \varphi$, from which we obtain $T h(\mathcal{N}) \cap \mathcal{L} \subseteq T \cap \mathcal{L}$. But $T \subseteq T h(\mathcal{N})$, consequently $T \cap \mathcal{L}=T h(\mathcal{N}) \cap \mathcal{L}$, and since $T$ is stable, from Proposition 3.7 we can conclude $T=T h(\mathcal{N})$. Therefore $\mathcal{M}$ is a ground $\mathcal{C}$-intended model for $I$.

\subsection{Possible-world semantics}

The notion of ground $\mathcal{C}$-intended model defined above provides a first preference semantics for ground logics. However, the preference criterion is based on the syntactic notion of comparing the theorems in two Kripke models. In the following we define a true possibleworld semantics for ground logics, i.e. we define a notion of minimality of Kripke models which directly derives from the structure of the models.

First, we need to define a relation between Kripke models that differ only with respect to the accessibility relation.

Definition 3.14 Given two Kripke models $\mathcal{M}_{1}=\left\langle W_{1}, R_{1}, V_{1}\right\rangle$ and $\mathcal{M}_{2}=\left\langle W_{2}, R_{2}, V_{2}\right\rangle$, $\mathcal{M}_{2} \supset_{G} \mathcal{M}_{1}$ if $W_{1}=W_{2}, V_{1}=V_{2}$ and $R_{2} \supset R_{1}$.

Using the $\supset_{G}$ relation, we are now able to define a partial ordering relation on Kripke models.

Definition 3.15 Let $\mathcal{M}_{1}, \mathcal{M}_{2}$ be two Kripke models. Then $\mathcal{M}_{2} \sqsubset_{G} \mathcal{M}_{1}$ if there exists a Kripke model $\mathcal{M}$ such that:

1. $\mathcal{M}_{2} \supset_{G} \mathcal{M} \odot \mathcal{M}_{1}$;

2. there exists a world $w \in W_{2} \backslash W_{1}$ such that for each world $w^{\prime} \in W_{1}, V_{2}\left(w^{\prime}\right) \neq V_{2}(w)$.

The above notion of partial ordering among Kripke models can informally be explained as follows: $\mathcal{M}_{2}$ is preferred to $\mathcal{M}_{1}$ if $\mathcal{M}_{2}$ is built starting from $\mathcal{M}_{1}$, by adding in front of it at least one world whose corresponding interpretation is different from those contained in $\mathcal{M}_{1}$, in such a way that each new world must be connected to all the worlds belonging to $\mathcal{M}_{1}$. Moreover, connections between worlds belonging to $\mathcal{M}_{1}$ and the new worlds are allowed.

Finally, minimal models are characterized using the $\sqsubset_{G}$ ordering, as a special case of Shoham's preference semantics [32].

Definition 3.16 Given a normal modal logic $\mathcal{S}$ characterized by the class of Kripke models $\mathcal{C}$, a model $\mathcal{M} \in \mathcal{C}$ is a ground $\mathcal{C}$-minimal model for $I$ if $\mathcal{M} \models I$ and for every model $\mathcal{M}^{\prime} \in \mathcal{C}$ such that $\mathcal{M}^{\prime}=I, \mathcal{M}^{\prime} \not \check{C}_{G} \mathcal{M}$.

The preference criterion obtained through the relation $\sqsubset_{G}$ can be seen as a stronger version of the minimality criterion found by Schwarz for McDermott and Doyle's logics [30]. The idea is the following: roughly speaking, Schwarz compares the S5-model $\mathcal{M}$ with all $\mathcal{S}$-models $\mathcal{N}$ such that $\mathcal{N}=\mathcal{M}^{\prime} \odot \mathcal{M}$, therefore $\mathcal{N}$ is such that

1. every world of $\mathcal{M}^{\prime}$ is connected to every world in $\mathcal{M}$; 
2. no world in $\mathcal{M}$ is connected to any world in $\mathcal{M}^{\prime}$.

The difference between Schwarz's ordering relation and $\sqsubset_{G}$ is that in the ground case the second condition does not hold, therefore connections between worlds in $\mathcal{M}^{\prime}$ and worlds in $\mathcal{M}$ are allowed (this is the intuitive meaning of $\mathcal{N} \supset_{G} \mathcal{M}^{\prime} \odot \mathcal{M}$ ).

Therefore, when checking for the minimality of a model $\mathcal{M}$, the ground criterion allows more models of the theory to be compared with $\mathcal{M}$. Hence, every model which is minimal in the class of models $\mathcal{C}$ according to the ground criterion, is minimal in $\mathcal{C}$ according to Schwarz's criterion as well, while the converse in general does not hold.

In the rest of this section we show the correspondence between the notion of ground expansion and the semantic notion of ground-minimal model, which in turn establishes a preference semantics for ground nonmonotonic modal logics. To prove such a correspondence, we need the following lemmata.

Lemma 3.17 Let $\mathcal{N}$ be a Kripke model and $\mathcal{M}$ be an S5-model such that:

1. $\mathcal{N} \models\{\neg K \varphi \mid \varphi \in \mathcal{L} \backslash T h(\mathcal{M})\} ;$

2. $T h(\mathcal{N}) \cap \mathcal{L}=T h(\mathcal{M}) \cap \mathcal{L}$.

Then, $\operatorname{Th}(\mathcal{N})=T h(\mathcal{M})$.

Proof The proof easily follows from the fact that $T h(\mathcal{M})$ is a stable theory and from Proposition 3.7 .

Lemma 3.18 Let $\mathcal{M}^{\prime}, \mathcal{M}^{\prime \prime}$ be S5-models. If $T h\left(\mathcal{M}^{\prime}\right) \cap \mathcal{L}=T h\left(\mathcal{M}^{\prime \prime}\right) \cap \mathcal{L}$ then $T h(\mathcal{N} \odot$ $\left.\mathcal{M}^{\prime}\right)=T h\left(\mathcal{N} \odot \mathcal{M}^{\prime \prime}\right)$ for any Kripke model $\mathcal{N}$.

Proof Since $\mathcal{M}^{\prime}$ and $\mathcal{M}^{\prime \prime}$ are S5-models, it follows that $T h\left(\mathcal{M}^{\prime}\right)$ and $T h\left(\mathcal{M}^{\prime \prime}\right)$ are stable theories, hence $T h\left(\mathcal{M}^{\prime}\right) \cap \mathcal{L}=T h\left(\mathcal{M}^{\prime \prime}\right) \cap \mathcal{L}$ implies $T h\left(\mathcal{M}^{\prime}\right)=T h\left(\mathcal{M}^{\prime \prime}\right)$, which in turn implies $\operatorname{Th}\left(\mathcal{N} \odot \mathcal{M}^{\prime}\right)=T h\left(\mathcal{N} \odot \mathcal{M}^{\prime \prime}\right)$ (see [20, Lemma 9.19]).

Finally, we state the equivalence between the syntactic and semantic characterizations defined above. This equivalence is proved under the restriction of cluster-decomposable classes of Kripke models.

Theorem 3.19 Given a normal modal logic $\mathcal{S}$, characterized by a cluster-decomposable class of Kripke models $\mathcal{C}$, a theory $I \subseteq \mathcal{L}_{K}$ and a stable theory $T$, let $\mathcal{M}$ be the canonical model for $T$. Then $T$ is an $\mathcal{S}_{G}$-expansion for $I$ if and only if $\mathcal{M}$ is a ground $\mathcal{C}$-minimal model for $I$.

Proof The proof makes use of the intermediate semantic notion of ground-intended model previously defined. From Theorem 3.13 it follows that theory $T$ is an $\mathcal{S}_{G}$-expansion for $I$ if and only if $\mathcal{M}$ is a ground $\mathcal{C}$-intended model for $I$, hence we only have to show that the model $\mathcal{M}$ is ground $\mathcal{C}$-intended for $I$ if and only if $\mathcal{M}$ is a ground $\mathcal{C}$-minimal model for $I$.

Only-if part. Assume $\mathcal{M}=\langle W, R, V\rangle$ is ground $\mathcal{C}$-intended for $I$. Then $\mathcal{M} \models I$ and for any $\mathcal{M}^{\prime} \in \mathcal{C}$, if $\mathcal{M}^{\prime} \models I \cup\{\neg K \varphi \mid \varphi \in \mathcal{L} \backslash T h(\mathcal{M})\}$, then $T h(\mathcal{M})=T h\left(\mathcal{M}^{\prime}\right)$. Now, suppose $\mathcal{M}$ is not ground $\mathcal{C}$-minimal for $I$. Then, there exists a Kripke model $\mathcal{N}=$ $\left\langle W_{N}, R_{N}, V_{N}\right\rangle \in \mathcal{C}$ such that $\mathcal{N} \mid=I$ and $\mathcal{N} \sqsubset_{G} \mathcal{M}$. This last fact implies $\mathcal{N} \supset_{G} \mathcal{N}^{\prime} \odot \mathcal{M}$ for some $\mathcal{N}^{\prime}$, i.e. every world of $\mathcal{N}$ is connected to every world of $\mathcal{M}$, consequently $\mathcal{N} \models$ $\{\neg K \varphi \mid \varphi \in \mathcal{L} \backslash T h(\mathcal{M})\}$. 
Since $\mathcal{M}$ is ground $\mathcal{C}$-intended for $I$ and $\mathcal{N} \models I$, from the above fact it follows that $T h(\mathcal{M})=T h(\mathcal{N})$, hence

$$
T h(\mathcal{M}) \cap \mathcal{L}=T h(\mathcal{N}) \cap \mathcal{L}
$$

On the other hand, since $\mathcal{N} \sqsubset_{G} \mathcal{M}$, Condition 2 of Def. 3.15 implies that there exists a world $w \in W_{N}$ such that $V_{N}(w) \neq V\left(w^{\prime}\right)$ for each $w^{\prime} \in W$. Now, if $V_{N}(w)$ satisfied every formula in the set $T h(\mathcal{M}) \cap \mathcal{L}$, then such a valuation would belong to $\mathcal{M}$, because $\mathcal{M}$ is the canonical model for $T=T h(\mathcal{M})$. Therefore $V_{N}(w)$ does not satisfy at least one formula of the set $T h(\mathcal{M}) \cap \mathcal{L}$, consequently $T h(\mathcal{M}) \cap \mathcal{L} \neq T h(\mathcal{N}) \cap \mathcal{L}$, which contradicts (3). Therefore, $\mathcal{M}$ must be a ground $\mathcal{C}$-minimal model for $I$, which proves the first part of the theorem.

If part. Assume $\mathcal{M}=\langle W, R, V\rangle$ is ground $\mathcal{C}$-minimal for $I$, i.e. $\mathcal{M} \models I$ and for any $\mathcal{M}^{\prime} \in \mathcal{C}$, if $\mathcal{M}^{\prime} \models I$, then $\mathcal{M}^{\prime} \not \subset G \mathcal{M}$. Then, suppose $\mathcal{M}$ is not ground $\mathcal{C}$-intended for $I$, i.e. there exists a model $\mathcal{N}=\left\langle W_{N}, R_{N}, V_{N}\right\rangle \in \mathcal{C}$ such that $\mathcal{N} \models I \cup\{\neg K \varphi \mid \varphi \in \mathcal{L} \backslash T h(\mathcal{M})\}$ and $T h(\mathcal{M}) \neq T h(\mathcal{N})$. Since $\mathcal{N} \models\{\neg K \varphi \mid \varphi \in \mathcal{L} \backslash T h(\mathcal{M})\}$, it follows that if $\varphi \in$ $T h(\mathcal{N}) \cap \mathcal{L}$, then $\varphi \in T h(\mathcal{M}) \cap \mathcal{L}$ (otherwise $\mathcal{N}=\neg K \varphi)$, hence $T h(\mathcal{N}) \cap \mathcal{L} \subseteq T h(\mathcal{M}) \cap \mathcal{L}$. Now, it cannot be the case that $T h(\mathcal{N}) \cap \mathcal{L}=T h(\mathcal{M}) \cap \mathcal{L}$, otherwise by Lemma 3.17 we would conclude $T h(\mathcal{N})=T h(\mathcal{M})$, which contradicts the hypothesis. Therefore,

$$
T h(\mathcal{N}) \cap \mathcal{L} \subset T h(\mathcal{M}) \cap \mathcal{L}
$$

Now, the class $\mathcal{C}$ is cluster-decomposable, therefore there exists a model $\mathcal{N}^{\prime}$ and an S5model $\mathcal{M}^{\prime}$ such that $\mathcal{N}=\mathcal{N}^{\prime} \odot \mathcal{M}^{\prime}$. Since $\mathcal{N} \models I \cup\{\neg K \varphi \mid \varphi \in \mathcal{L} \backslash T h(\mathcal{M})\}$ and $\mathcal{N}=\mathcal{N}^{\prime} \odot \mathcal{M}^{\prime}$, it follows that

$$
\mathcal{M}^{\prime} \models I \cup\{\neg K \varphi \mid \varphi \in \mathcal{L} \backslash T h(\mathcal{M})\}
$$

This in turn implies $T h\left(\mathcal{M}^{\prime}\right) \cap \mathcal{L} \subseteq T h(\mathcal{M}) \cap \mathcal{L}$, otherwise there would exist a formula $\varphi \in \mathcal{L}$ such that $\varphi \in T h\left(\mathcal{M}^{\prime}\right)$ and $\varphi \notin T h(\mathcal{M})$. Now, since $\mathcal{M}^{\prime}$ is an S5-model, by Proposition 3.3 it follows that $T h\left(\mathcal{M}^{\prime}\right)$ is a stable theory. Hence $\neg K \varphi \notin T h\left(\mathcal{M}^{\prime}\right)$, thus contradicting (5).

Now, there are two possible cases:

1. $T h\left(\mathcal{M}^{\prime}\right) \cap \mathcal{L} \subset T h(\mathcal{M}) \cap \mathcal{L}$. In this case $T h\left(\mathcal{M}^{\prime}\right)$ is a stable theory which has a smaller objective part than $T=T h(\mathcal{M})$. Moreover, (5) implies that $T h\left(\mathcal{M}^{\prime}\right)$ contains $I$, thus contradicting the hypothesis that $T$ is an $S 5_{G}$-expansion for $I$ (see Proposition 2.2);

2. $T h\left(\mathcal{M}^{\prime}\right) \cap \mathcal{L}=T h(\mathcal{M}) \cap \mathcal{L}$. This, together with (4) implies $T h\left(\mathcal{N}^{\prime}\right) \cap \mathcal{L} \subset T h(\mathcal{M}) \cap \mathcal{L}$, which in turn implies that the model $\mathcal{N}^{\prime}=\left\langle V_{N}^{\prime}, R_{N}^{\prime}, W_{N}^{\prime}\right\rangle$ contains at least one world $w$ such that $V_{N}^{\prime}(w) \neq V\left(w^{\prime}\right)$ for each world $w^{\prime}$ in $\mathcal{M}$. Consequently, the model $\mathcal{M}^{\prime \prime}=\mathcal{N}^{\prime} \odot \mathcal{M}$ is such that $\mathcal{M}^{\prime \prime} \sqsubset_{G} \mathcal{M}$. Moreover, since $\mathcal{C}$ is cluster-decomposable, it follows that $\mathcal{M}^{\prime \prime} \in \mathcal{C}$. Finally, Lemma 3.18 implies that $T h(\mathcal{N})=T h\left(\mathcal{M}^{\prime \prime}\right)$, hence $\mathcal{M}^{\prime \prime} \models I$. From the three above facts it follows that $\mathcal{M}$ is not a ground $\mathcal{C}$-minimal model for $I$, thus contradicting the hypothesis.

Therefore, $\mathcal{M}$ must be a ground $\mathcal{C}$-intended model for $I$, which proves the second part of the theorem.

The above theorem provides a semantic characterization for a subset of the family of ground nonmonotonic logics $\mathcal{S}_{G}$, since it relates the solutions of Equation (2) to groundminimal models. In particular, the correspondence is shown to hold for the ground logics built from modal logics S5, KD45, S4F, SW5. 


\section{Reasoning}

After providing a semantic account of ground logics, we now address reasoning in these logics. Since we are studying ground logics as a knowledge representation formalism, we focus our analysis on logical entailment $\mid=\mathcal{S}_{G}$, which is usually considered the basis for the realization of the deductive services of a knowledge-based system.

Moreover, from the knowledge representation perspective it is interesting to look at the computational adequacy of reasoning procedures. This requires not only to provide algorithms for computing entailment in ground logics, but also to characterize its computational complexity. To this end, we briefly recall some basic notions from complexity theory (see [12]). We denote as $\mathrm{P}$ the class of problems solvable in polynomial time by a deterministic Turing machine. The class NP contains all problems that can be solved by a nondeterministic Turing machine in polynomial time. The class coNP comprises all problems that are the complement of a problem in NP. A problem $P_{1}$ is said to be NP-complete if it is in NP and for every problem $P_{2}$ in NP, there is a polynomial-time reduction from $P_{2}$ to $P_{1}$. If there is a polynomial-time reduction from an NP-complete problem $P_{2}$ to a problem $P_{1}$, then $P_{1}$ is said to be NP-hard. With a slight abuse of terminology, we call NP-algorithm a nondeterministic algorithm that runs in polynomial time. $\mathrm{P}^{A}\left(\mathrm{NP}^{A}\right)$ is the class of problems that are solved in polynomial time by deterministic (nondeterministic) Turing machines using an oracle for $A$ (i.e. that solves in constant time any problem in $A$ ). Finally, the classes $\Sigma_{k}^{p}, \Pi_{k}^{p}$ and $\Delta_{k}^{p}$ of the polynomial hierarchy $(\mathrm{PH})$ are defined by $\Sigma_{0}^{p}=\Pi_{0}^{p}=\Delta_{0}^{p}=\mathrm{P}$, and for $k \geq 0$, $\Sigma_{k+1}^{p}=\mathrm{NP}^{\Sigma_{k}^{p}}, \Pi_{k+1}^{p}=\operatorname{co} \Sigma_{k+1}^{p}$ and $\Delta_{k+1}^{p}=\mathrm{P}^{\Sigma_{k}^{p}}$.

The complexity analysis of logical entailment in ground logics is developed as follows. We first provide lower bounds: we prove that entailment in $\mathrm{S}_{G}$ is $\Pi_{3}^{p}$-hard, and extend this result to the other ground logics. Secondly, we provide upper bounds: we give a finitary characterization of $\mathcal{S}_{M D D}$-expansions, then we show that verifying that an $\mathcal{S}_{M D D}$-expansion is not ground is a problem in $\Sigma_{2}^{p}$ for logics S5, KD45, S4F, SW5, and finally we give a nondeterministic algorithm for non-entailment which uses the above check as an oracle. This proves that non-entailment is in $\Sigma_{3}^{p}$, hence entailment is $\Pi_{3}^{p}$-complete for ground logics $\mathrm{S} 5_{G}, \mathrm{KD} 45_{G}, \mathrm{~S} 4 \mathrm{~F}_{G}, \mathrm{SW} 5_{G}$, thus extending the results presented in [5] for $\mathrm{KD} 45_{G}$.

\subsection{Lower bounds}

Below we provide lower bounds for logical entailment in ground logics by reducing the $\Sigma_{3}^{p}$ complete problem of deciding validity of $\exists \forall \exists$-quantified boolean formulae ( $\exists \forall \exists-\mathrm{QBF}$ ) to non-entailment. We first address the case of $\mathrm{S} 5_{G}$, then extend the result to $\mathrm{KD} 45_{G}, \mathrm{~S} 4 \mathrm{~F}_{G}, \mathrm{SW} 5_{G}$. Let $\varphi$ be a propositional formula over $\mathcal{L}=\left\{x_{1}, \ldots, x_{n}, y_{1}, \ldots, y_{m}, z_{1}, \ldots, z_{l}\right\}$, and let $F$ be the $\exists \forall \exists-\mathrm{QBF}$

$$
\exists x_{1} \ldots \exists x_{n} \forall y_{1} \ldots \forall y_{m} \exists z_{1} \ldots \exists z_{l} \cdot \varphi
$$

It is well known that deciding validity of such formulae is a $\Sigma_{3}^{p}$-complete problem [12].

Theorem 4.1 Entailment in $\mathrm{S}_{G}$ is $\Pi_{3}^{p}$-hard.

Proof Let $p$ be a new atom which we add to $\mathcal{L}$. We show that $F$ is valid if and only if $I \forall \forall_{\mathrm{S}_{G}} \neg K p$, where $I$ contains the three formulae

$$
I_{1}=\bigwedge_{i=1}^{n}\left(K x_{i} \vee K \neg x_{i}\right)
$$




$$
\begin{aligned}
I_{2} & =\left(K p \supset \bigwedge_{j=1}^{m} y_{j}\right) \\
I_{3} & =(K p \vee \neg \psi)
\end{aligned}
$$

and $\psi$ is the formula (over $\mathcal{L}_{K}$ ) obtained by replacing each $y_{j}$ with $K y_{j}$ in $\varphi$. Clearly, $I \not \forall_{\mathrm{S} 5_{G}} \neg K p$ iff there exists an $\mathrm{S}_{G}$-expansion $T$ for $I$ such that $p \in T$. Moreover, since $I_{2} \in T$, such a $T$ must also contain $y_{1}, \ldots, y_{m}$. Finally, since $I_{1} \in T$, for each $i=1 . . n$ either $x_{i} \in T$ or $\neg x_{i} \in T$. Hence $I \not \forall_{\mathrm{S}_{G}} \neg K p$ iff there exists an $\mathrm{S}_{G}$-expansion containing $p, y_{1}, \ldots, y_{m}$, and for $i=1 . . n$ exactly one between $x_{i}, \neg x_{i}$.

From the definition of validity for QBF, $F$ is valid iff there exists a (partial) propositional valuation $v$ to the atoms $x_{1}, \ldots, x_{n}$ such that the formula

$$
F_{v}=\forall y_{1} \ldots \forall y_{m} \exists z_{1} \ldots \exists z_{l} \cdot \varphi_{v}
$$

is valid. Define the stable expansion $T(v)=S T\left(\left\{x_{i} \mid v\left(x_{i}\right)=\operatorname{true}\right\} \cup\left\{\neg x_{i} \mid v\left(x_{i}\right)=\right.\right.$ false $\left.\} \cup\left\{p, y_{1}, \ldots, y_{m}\right\}\right)$. Clearly, $I \subseteq T(v)$.

We show that $F$ is valid iff there exists a $v$ such that $T(v)$ is an $\mathrm{S}_{G}$-expansion for $I$.

Only-if part. First, suppose $F$ is valid, i.e., there exists a $v$ such that $F_{v}$ is valid. We show that $T(v)$ is ground for $I$, i.e. there does not exist a stable theory $S$ containing $I$ such that $S \cap \mathcal{L} \subset T(v) \cap \mathcal{L}$. In fact, suppose such a theory $S$ exists. Then, since $S$ contains $I_{1}$, either $x_{i}$ or $\neg x_{i}$ is in $S$, for $i=1 . . n$. Since $S \cap \mathcal{L} \subset T(v) \cap \mathcal{L}$, for each $i=1 . . n, x_{i} \in S$ iff $x_{i} \in T(v)$ and $\neg x_{i} \in S$ iff $\neg x_{i} \in T(v)$. Moreover, $p \notin S$, otherwise it would follow that $y_{1}, \ldots, y_{m} \in S$, therefore $S \cap \mathcal{L} \not \subset T(v) \cap \mathcal{L}$, contradicting the hypothesis.

Since $S$ is stable, $p \notin S$ implies $\neg K p \in S$ hence $\neg \psi \in S$. Since for each $i=1$..n, $x_{i} \in S$ iff $v\left(x_{i}\right)=$ true and $\neg x_{i} \in S$ iff $v\left(x_{i}\right)=$ false, also $(\neg \psi)_{v} \in S$. Let $u$ be the partial valuation over $\left\{y_{1}, \ldots, y_{m}\right\}_{K}$ such that:

- $u\left(y_{i}\right)=u\left(K y_{j}\right)=$ true iff $y_{i} \in S$ (hence iff $K y_{j} \in S$ by stability of $S$ );

- $u\left(y_{i}\right)=u\left(K y_{j}\right)=$ false iff $y_{i} \notin S$ (in this case, $\left.\neg K y_{j} \in S\right)$.

Since $(\neg \psi)_{v} \in S$, also $\left((\neg \psi)_{v}\right)_{u} \in S$. Now observe that $\left((\neg \psi)_{v}\right)_{u}$ is an objective formula over $\left\{z_{1}, \ldots, z_{l}\right\}$. Moreover, since $T(v)$ contains no theorems over $\left\{z_{1}, \ldots, z_{l}\right\}$ but tautologies, also $S$ does not, therefore $\left((\neg \psi)_{v}\right)_{u}$ is a tautology; this implies that the formula $\left(\psi_{v}\right)_{u}$ is unsatisfiable.

By hypothesis, $F_{v}$ is valid, hence considering the above defined valuation $u$ over $\left\{y_{1}, \ldots, y_{m}\right\}_{K}$, formula $\exists z_{1} \ldots \exists z_{l}\left(\varphi_{v}\right)_{u}$ is valid - that is, the propositional formula $\left(\varphi_{v}\right)_{u}$ over $\left\{z_{1}, \ldots, z_{l}\right\}$ is satisfiable. Now observe that $u$ assigns the same truth values to $y_{j}$ and $K y_{j}$, for $j=1 . . m$, hence $\left(\varphi_{v}\right)_{u}$ and $\left(\psi_{v}\right)_{u}$ are the same formula, which should be both satisfiable and unsatisfiable. Therefore, such an $S$ does not exists, and $T(v)$ is an $\mathrm{S} 5_{G}$-expansion.

If part. Suppose $F$ is not valid. Then, for every valuation $v$ over $x_{1}, \ldots x_{n}, F_{v}$ is not valid, hence formula $\neg F_{v}$ is valid, where $\neg F_{v}$ can be rewritten as

$$
\exists y_{1} \ldots \exists y_{m} \forall z_{1} \ldots \forall z_{l} \cdot \neg \varphi_{v}
$$

Hence, for every $v$ there exists an assignment $u$ to $y_{1}, \ldots, y_{m}$ such that $\forall z_{1} \ldots \forall z_{l} \bullet\left(\neg \varphi_{v}\right)_{u}$ is valid-that is, the propositional formula $\left(\neg \varphi_{v}\right)_{u}$ over $\left\{z_{1}, \ldots, z_{l}\right\}$ is a tautology.

We show that $T(v)$ is not an $\mathrm{S} 5_{G}$-expansion for $I$. Define the stable expansion $S(v)=$ $S T\left(\left\{x_{i} \mid v\left(x_{i}\right)=\right.\right.$ true $\} \cup\left\{\neg x_{i} \mid v\left(x_{i}\right)=\right.$ false $\} \cup\left\{y_{i} \mid u\left(y_{i}\right)=\right.$ true $\left.\}\right)$. Observe that 
$S(v) \cap \mathcal{L} \subset T(v) \cap \mathcal{L}$. Hence this part is proven if $I \subseteq S(v)$. In fact, $I_{1} \in S(v)$, and since $p \notin S(v)$, then $\neg K p \in S(v)$, then $I_{2} \in S(v)$.

Extend the partial valuation $u$ in this way:

- $u\left(K y_{j}\right)=$ true iff $y_{i} \in S(v)$ (hence iff $u\left(y_{i}\right)=$ true);

- $u\left(K y_{j}\right)=$ false iff $\neg K y_{j} \in S(v)$ (in this case, $u\left(y_{i}\right)=$ false).

Now observe that $\left((\neg \psi)_{v}\right)_{u}$ and $\left(\neg \varphi_{v}\right)_{u}$ are the same formula, hence $\left((\neg \psi)_{v}\right)_{u}$ is a tautology, hence $\left((\neg \psi)_{v}\right)_{u} \in S(v)$. But from the definition of $v$ and $u,\left((\neg \psi)_{v}\right)_{u} \in S(v)$ iff $\neg \psi \in S(v)$. Therefore, also $I_{3} \in S(v)$. We conclude that for every $v, T(v)$ is not an $S 5_{G}$-expansion for $I$, which concludes the proof.

We now extend the above result to the other ground logics.

Theorem 4.2 Given a modal logic $\mathcal{S}$ such that $\mathrm{K} \subseteq \mathcal{S} \subseteq \mathrm{S} 5$ and $\mathcal{S}$ satisfies the terminal cluster property, entailment in $\mathcal{S}_{G}$ is $\Pi_{3}^{p}$-hard.

Proof For each logic $\mathcal{S}$, the reduction follows the one given for $\mathrm{S} 5_{G}$.

Given an $\exists \forall \exists$-QBF formula $F=\exists x_{1} \ldots \exists x_{n} \forall y_{1} \ldots \forall y_{m} \exists z_{1} \ldots \exists z_{l} . \varphi$, we define $I^{\prime}$ as the set containing $I_{1}, I_{2}, I_{3}$, defined as in (6), (7), (8) respectively, and the formulae

$$
\begin{aligned}
I_{4} & =\bigwedge_{i=1}^{n} K x_{i} \supset x_{i} \\
I_{5} & =\bigwedge_{i=1}^{n} K \neg x_{i} \supset \neg x_{i} \\
I_{6} & =K p \supset p \\
I_{7} & =\neg K \neg K p \supset K p
\end{aligned}
$$

Then we show that $F$ is valid iff $I^{\prime} \not \nvdash_{\mathcal{S}_{G}} \neg K p$.

If part. By contraposition: if $F$ is not valid, from the previous theorem no $\mathrm{S}_{G}$-expansion for $I=\left\{I_{1}, I_{2}, I_{3}\right\}$ contains $p$. Since $I_{4}, I_{5}, I_{6}$ and $I_{7}$ are theorems in S5, it follows that $I^{\prime}$ is $\mathrm{S} 5$-equivalent to $I$, in the sense that $I^{\prime}$ and $I$ are satisfied by the same set of S5 models. Consequently, from Proposition 3.6, $\mathcal{S}_{G}$-expansions for $I^{\prime}$ are a subset of the set of $\mathrm{S}_{G^{-}}$ expansions for $I$, and therefore $I^{\prime} \models \mathcal{S}_{G} \neg K p$.

Only-if part. Suppose $F$ is valid. Define both $v$ (a particular propositional valuation to $\left.x_{1}, \ldots, x_{n}\right)$ and $T(v)$ as in the proof of the previous theorem. Let $\mathcal{M}=\langle W, R, V\rangle$ be the canonical model for $T(v)$ and $\mathcal{C}$ be the class of models characterizing logic $\mathcal{S}$, and suppose $T(v)$ is not an $\mathcal{S}_{G}$-expansion for $I^{\prime}$. Then, from Theorem $3.13, \mathcal{M}$ is not ground $\mathcal{C}$-intended for $I^{\prime}$, which implies that there exists a model $\mathcal{N}$ in $\mathcal{C}$ such that

1. $\mathcal{N} \models I^{\prime} \cup\{\neg K \varphi \mid \varphi \in \mathcal{L} \backslash T h(\mathcal{M})\}$;

2. $T h(\mathcal{N}) \neq T h(\mathcal{M})$.

we divide the rest of the proof in four parts:

(A) First of all, we show that for $i=1, \ldots, n$ if $\mathcal{M} \models x_{i}$ then $\mathcal{N} \models x_{i}$. In fact, $\mathcal{M} \models x_{i}$ implies $\mathcal{M} \not \models \neg x_{i}$ implies (from Condition 1.) $\mathcal{N} \models \neg K \neg x_{i}$. Since $\mathcal{N} \models I_{1}$, also $\mathcal{N} \models$ 
$K x_{i}$, and from $\mathcal{N} \models I_{4}$ one concludes that $\mathcal{N} \models x_{i}$. An analogous proof shows that for $i=1, \ldots, n$ if $\mathcal{M} \models \neg x_{i}$ then $\mathcal{N} \models \neg x_{i}$.

(B) Given any world $w$ of $\mathcal{N}$, let $Y$ be the terminal cluster for $w$ mentioned in Definition 3.8, and let $T h(Y)$ denote the formulae which are true in all worlds of $Y$. We prove that $T h(Y) \cap$ $\mathcal{L}=T h(\mathcal{M}) \cap \mathcal{L}$.

In fact, $T h(Y) \cap \mathcal{L} \subseteq T h(\mathcal{M}) \cap \mathcal{L}$ can be proven by contradiction: suppose there exists a formula $\varphi$ such that $\varphi \in T h(Y) \cap \mathcal{L}$ and $\varphi \notin T h(\mathcal{M}) \cap \mathcal{L}$; then from Condition 2. of

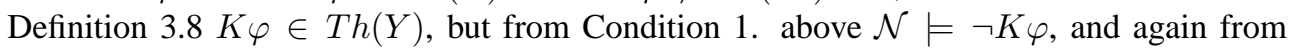
Condition 2. of Definition 3.8 it follows $\neg K \varphi \in T h(Y)$, which is a contradiction. Then $T h(Y) \cap \mathcal{L} \not \subset T h(\mathcal{M}) \cap \mathcal{L}$ follows from the fact that $T(v)=T h(\mathcal{M})$ is an $S 5_{G}$-expansion for $I$, hence there can not exist an S5-model $\left\langle Y, Y \times Y, V_{N}\right\rangle$ with fewer objective theorems. Therefore, $T h(Y) \cap \mathcal{L}=T h(\mathcal{M}) \cap \mathcal{L}$. Observe that this together with the fact that $Y$ is a terminal cluster implies that $T h(Y)=T h(\mathcal{M})$.

(C) Then we show that $\mathcal{N} \models p$ :

Since $p \in T h(\mathcal{M}) \cap \mathcal{L}$, also $p \in T h(Y) \cap \mathcal{L}$, and since $Y$ is a terminal cluster also $K p \in$ $T h(Y)$. Hence $\langle\mathcal{N}, w\rangle \models \neg K \neg K p$. Since $w$ is arbitrary, we conclude that $\mathcal{N} \models \neg K \neg K p$. Now from $I_{7}$ and $I_{6}$ we derive that $\mathcal{N} \models p$.

(D) Finally, we prove that $\mathcal{N} \models p$ contradicts Condition 2. above:

In fact, from $\mathcal{N}=I_{2}$ and part (A) above it follows that $T h(\mathcal{M}) \cap \mathcal{L} \subseteq T h(\mathcal{N}) \cap \mathcal{L}$. Now $T h(\mathcal{M}) \cap \mathcal{L} \not \subset T h(\mathcal{N}) \cap \mathcal{L}$ can proved by contradiction: if $\varphi \in T h(\mathcal{N}) \cap \mathcal{L}$ and $\varphi \notin T h(\mathcal{M}) \cap \mathcal{L}$, then from necessitation $K \varphi \in T h(\mathcal{N})$, but from Condition 1. above $\mathcal{N} \models$ $\neg K \varphi$, which is a contradiction. Combining these two facts yields $T h(\mathcal{M}) \cap \mathcal{L}=T h(\mathcal{N}) \cap \mathcal{L}$.

Therefore, each valuation of a world in $\mathcal{N}$ coincides with some valuation of a world in $\mathcal{M}$. Moreover, from part (B) every world $w$ in $\mathcal{N}$ is connected to a cluster $Y$ having the same theorems as $\mathcal{M}$. These two facts imply $T h(\mathcal{N})=T h(\mathcal{M})$ thus contradicting Condition 2.

From (A)-(D), $T(v)$ is a $\mathcal{S}_{G}$-expansion for $I^{\prime}$, consequently $I^{\prime} \nvdash_{\mathcal{S}_{G}} \neg K p$.

The above theorems show that in many cases reasoning in ground logics is harder than in McDermott and Doyle's logics. In the following subsection we show that this fact is due to an additional step which makes a selection over $\mathcal{S}_{M D D}$-expansions.

\subsection{Upper bounds}

Now we consider the upper bounds of complexity of reasoning in ground logics. Clearly, proving $I \not \mathcal{S}_{G} \varphi$ amounts to exhibiting an $\mathcal{S}_{G}$-expansion for $I$ which does not contain $\varphi$. Hence, the first point is to give a finitary characterization of ground expansions. This is done by restricting the attention to all the subformulae of $I$. Let $I \subseteq \mathcal{L}_{K}$. We denote with $M A(I)$ the following set of formulae: $M A(I)=\{\varphi \mid K \varphi$ is a subformula of a formula from $I\}$. Moreover, we say that a modal formula $\varphi$ is an I-formula if it is a formula built from propositional variables of $\mathcal{L}$ and from modal atoms of the form $K \psi$, where $\psi \in M A(I)$.

Then, following [20], we introduce the notion of introspection-consistency on pairs whose elements are subsets of $M A(I)$.

Definition 4.3 Let $I \subseteq \mathcal{L}_{K}$. A pair $(\Phi, \Psi)$ of subsets of $M A(I)$ is called introspectionconsistent with $I$ iff the following conditions hold: 
1. $\Phi \cup \Psi=M A(I)$ and $\Phi \cap \Psi=\emptyset$ (i.e. $(\Phi, \Psi)$ is a partition of $M A(I)$ );

2. $I \cup \Psi \cup\{\neg K \varphi \mid \varphi \in \Phi\} \cup\{K \varphi \mid \varphi \in \Psi\}$ is propositionally consistent;

3. for each $\varphi \in \Phi, \varphi \notin C n(I \cup \Psi \cup\{\neg K \varphi \mid \varphi \in \Phi\} \cup\{K \varphi \mid \varphi \in \Psi\})$.

Note that since only propositional consistency and non-implication are involved in the above definition, introspection consistency can be decided with a polynomial number of calls to an NP-oracle. Note also that a partition of $M A(I)$ has polynomial size wrt $I$.

The idea is to identify particular introspection-consistent partitions, that are appropriate to give a finite characterization of ground expansions. In order to study the relationship between introspection-consistent partitions and ground logics, we need to address the relationship between partitions and with stable theories. To this end, we recall the following definitions.

Definition 4.4 Let $I \subseteq \mathcal{L}_{K}$ and let the partition $(\Phi, \Psi)$ of $M A(I)$ be introspection-consistent with $I$. Then:

1. we call $M_{I}(\Phi, \Psi)$ the set of all the propositional valuations $v$ of $\mathcal{L}_{K}$ satisfying $I \cup \Psi \cup$ $\{\neg K \varphi \mid \varphi \in \Phi\} \cup\{K \varphi \mid \varphi \in \Psi\})$;

2. for a valuation $v \in M_{I}(\Phi, \Psi)$ we call $U(\Phi, \Psi)(v)$ a propositional valuation of $\mathcal{L}$ such that $U(\Phi, \Psi)(v)=v_{\mid \mathcal{L}}$;

3. an S5-model $\mathcal{M}_{I}(\Phi, \Psi)=\left\langle M_{I}(\Phi, \Psi), U(\Phi, \Psi)\right\rangle$ is called canonical for the partition $(\Phi, \Psi)$

4. the theory $\operatorname{Th}\left(\mathcal{M}_{I}(\Phi, \Psi)\right)$ is called canonical for $(\Phi, \Psi)$ and is denoted by $T_{I}(\Phi, \Psi)$.

Proposition 4.5 [20, 9.36] Let $I \subseteq \mathcal{L}_{K}$ and let $T \subseteq \mathcal{L}_{K}$ be a stable and consistent theory containing $I$. Then the partition $(M A(I) \backslash T, M A(\bar{I}) \cap T)$ is introspection-consistent with $I$.

Proposition 4.6 [20, 9.39] Let $I \subseteq \mathcal{L}_{K}$. If a partition $(\Phi, \Psi)$ of $M A(I)$ is introspectionconsistent with $I$, then:

1. the theory $T_{I}(\Phi, \Psi)$ is stable;

2. for every $I$-formula $\varphi$ and for every world $v \in M_{I}(\Phi, \Psi),\left\langle\mathcal{M}_{I}(\Phi, \Psi), v\right\rangle=\varphi$ iff $v(\varphi)=$ true;

3. $I \cup \Psi \cup\{\neg K \varphi \mid \varphi \in \Phi\} \cup\{K \varphi \mid \varphi \in \Psi\} \subseteq T_{I}(\Phi, \Psi)$;

4. $\Phi=M A(I) \backslash T_{I}(\Phi, \Psi)$ and $\Psi=M A(I) \cap T_{I}(\Phi, \Psi)$;

5. $T_{I}(\Phi, \Psi)=S T(C n(I \cup \Psi \cup\{\neg K \varphi \mid \varphi \in \Phi\} \cup\{K \varphi \mid \varphi \in \Psi\}) \cap \mathcal{L})$.

We can now provide a finite characterization of ground expansions in terms of particular introspection-consistent partitions.

Theorem 4.7 Let $\mathcal{S}$ be a modal logic such that $\mathrm{K} \subseteq \mathcal{S} \subseteq$ S5. Let $T$ be an $\mathcal{S}_{G}$-expansion of a theory $I \subseteq \mathcal{L}_{K}$. Let $\Phi=M A(I) \backslash T, \Psi=M A(I) \cap T$. Then the partition $(\Phi, \Psi)$ is introspection-consistent with $I$ and $T=T_{I}(\Phi, \Psi)$. 
Proof Since $T$ is stable and consistent (Proposition 3.5), from Proposition 4.5 it follows that $(\Phi, \Psi)$ is introspection-consistent with $I$. Therefore we have to show that $T=T_{I}(\Phi, \Psi)$. Since $T_{I}(\Phi, \Psi)$ is also stable, it is sufficient to show that $T \cap \mathcal{L}=T_{I}(\Phi, \Psi) \cap \mathcal{L}$.

First, consider a formula $\psi \in T_{I}(\Phi, \Psi)$. Then, $\psi \in C n(I \cup \Psi \cup\{\neg K \varphi \mid \varphi \in \Phi\} \cup\{K \varphi \mid$ $\varphi \in \Psi\}$ ). By hypothesis, $I \subseteq T$. By definition of $\Phi$, if $\varphi \in \Phi$ then $\varphi \notin T$, consequently, by stability of $T, \neg K \varphi \in T$. Analogously, by definition of $\Psi$, if $\varphi \in \Psi$ then $\varphi \in T$, consequently, by stability of $T, K \varphi \in T$. Therefore $T \supseteq I \cup \Psi \cup\{\neg K \varphi \mid \varphi \in \Phi\} \cup\{K \varphi \mid$ $\varphi \in \Psi\}$, and since $T$ is stable, $T \supseteq C n(I \cup \Psi \cup\{\neg K \varphi \mid \varphi \in \Phi\} \cup\{K \varphi \mid \varphi \in \Psi\})$, hence $\psi \in T$.

Now suppose $\psi \in T \cap \mathcal{L}$. Then, $\psi \in C n_{\mathrm{S} 5}(I \cup\{\neg K \varphi \mid \varphi \in \mathcal{L} \backslash T\})$. From Point 3. in the previous Proposition 4.6, $I \subseteq T_{I}(\Phi, \Psi)$. Moreover, from the first part of this proof - by contraposition - if $\varphi \in \mathcal{L} \backslash T$ then $\varphi \notin T_{I}(\Phi, \Psi)$, and since $T_{I}(\Phi, \Psi)$ is stable (Point 1 . in the previous Proposition 4.6) $\neg K \varphi \in T_{I}(\Phi, \Psi)$. Therefore, $\{\neg K \varphi \mid \varphi \in \mathcal{L} \backslash T\} \subseteq T_{I}(\Phi, \Psi)$. Since $T_{I}(\Phi, \Psi)$ is closed under consequence in S5 [20, Theorem 8.4], $\psi \in T_{I}(\Phi, \Psi)$.

We point out that this result provides for a finite characterization of expansions for every ground logic. In this sense it strengthens an analogous result (see Prop. 4.9) obtained for MDD logics, which does not include e.g. the S5 case.

We can finally turn the correspondence between introspection-consistent partitions of $M A(I)$ and ground expansions into an actual characterization of the expansions of the ground logic $\mathrm{S} 5$, thus obtaining a finitary characterization for $\mathrm{S} 5_{G}$-expansions.

Theorem 4.8 Let $I \subseteq \mathcal{L}_{K}$. Let $(\Phi, \Psi)$ be a partition of $M A(I)$. Then, $T_{I}(\Phi, \Psi)$ is an

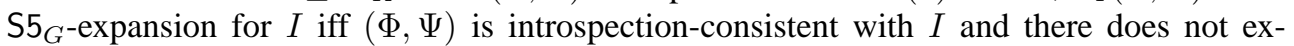
ist a partition $\left(\Phi^{\prime}, \Psi^{\prime}\right)$ of $M A(I)$ such that $\left(\Phi^{\prime}, \Psi^{\prime}\right)$ is introspection-consistent with $I$ and $T_{I}\left(\Phi^{\prime}, \Psi^{\prime}\right) \cap \mathcal{L} \subset T_{I}(\Phi, \Psi) \cap \mathcal{L}$.

Proof Only-if part. Suppose $T_{I}(\Phi, \Psi)$ is an $\mathrm{S} 5_{G}$-expansion for $I$. Then, from Theorem 4.7 it follows that $(\Phi, \Psi)$ is introspection-consistent with $I$. Now, suppose there exists a partition $\left(\Phi^{\prime}, \Psi^{\prime}\right)$ which is introspection-consistent with $I$ and such that $T_{I}\left(\Phi^{\prime}, \Psi^{\prime}\right) \cap \mathcal{L} \subset$ $T_{I}(\Phi, \Psi) \cap \mathcal{L}$. The existence of such a theory $T_{I}\left(\Phi^{\prime}, \Psi^{\prime}\right)$ contradicts Proposition 3.9, since by hypothesis $T_{I}(\Phi, \Psi)$ is an $\mathrm{S} 5_{G}$-expansion for $I$.

If part. Suppose $(\Phi, \Psi)$ is introspection-consistent with $I$ and there does not exist a partition $\left(\Phi^{\prime}, \Psi^{\prime}\right)$ of $M A(I)$ such that $\left(\Phi^{\prime}, \Psi^{\prime}\right)$ is introspection-consistent with $I$ and $T_{I}\left(\Phi^{\prime}, \Psi^{\prime}\right) \cap$ $\mathcal{L} \subset T_{I}(\Phi, \Psi) \cap \mathcal{L}$. Now, suppose that $T_{I}(\Phi, \Psi)$ is not an $\mathrm{S}_{G^{-}}$-expansion for $I$. Therefore, there exists a stable theory $T^{\prime}$ containing $I$ and such that $T^{\prime} \cap \mathcal{L} \subset T \cap \mathcal{L}$. By Proposition 4.5 the partition $\left(\Phi^{\prime}, \Psi^{\prime}\right)=\left(M A(I) \backslash T^{\prime}, M A(I) \cap T^{\prime}\right)$ is introspection-consistent with $I$. Moreover, Proposition 4.6 implies $T_{I}\left(\Phi^{\prime}, \Psi^{\prime}\right)=T^{\prime}$, consequently $T_{I}\left(\Phi^{\prime}, \Psi^{\prime}\right) \cap \mathcal{L} \subset T_{I}(\Phi, \Psi) \cap \mathcal{L}$, which contradicts the hypothesis.

The above theorem gives a (nondeterministic) method to show that, given an introspectionconsistent partition $(\Phi, \Psi)$ of $M A(I), T_{I}(\Phi, \Psi)$ is not an $\mathrm{S}_{G}$-expansion for $I$ : guess another partition $\left(\Phi^{\prime}, \Psi^{\prime}\right)$ of $M A(I)$, and verify that $\left(\Phi^{\prime}, \Psi^{\prime}\right)$ is introspection-consistent with $I$ and $T_{I}\left(\Phi^{\prime}, \Psi^{\prime}\right) \cap \mathcal{L} \subset T_{I}(\Phi, \Psi) \cap \mathcal{L}$. It can be shown [3] that both checks can be done with a polynomial (wrt the size of $I$ ) number of calls to an NP-oracle, hence the problem is in $\Sigma_{2}^{p}$.

We now want to extend this method to other ground logics. To this end, we exploit the following property. 
Proposition 4.9 [20, 9.41] Let $\mathcal{S}$ be a modal logic such that $\mathrm{K} \subseteq \mathcal{S} \subseteq \mathrm{KD} 45$ or $\mathrm{K} \subseteq \mathcal{S} \subseteq$ SW5. Let $I \subseteq \mathcal{L}_{K}$. If a theory $T \subseteq \mathcal{L}_{K}$ is an $\mathcal{S}_{M D D}$-expansion for $I$, then $(\Phi, \Psi)$, where $\Phi=M A(I) \backslash T$ and $\Psi=M A(I) \cap T$, is introspection-consistent with $I$ and $T=T_{I}(\Phi, \Psi)$.

We call EXPANSION $(\mathcal{S}, I,(\Phi, \Psi))$ an algorithm that given a modal logic $\mathcal{S}$ among S4F, KD45, SW5, a theory $I$ and a partition $(\Phi, \Psi)$ of $M A(I)$ introspection-consistent with $I$, checks whether $T_{I}(\Phi, \Psi)$ is an $\mathcal{S}_{M D D}$-expansion. It is known [20, Section 11.1] that for each of the three modal logics, EXPANSION can be computed by a polynomial-time algorithm which makes a polynoial number of calls to an NP-oracle. Since $\mathrm{S} 5_{M D D}$-expansions for $I$ correspond to stable theories containing $I$, we can extend EXPANSION also to the (trivial) case of $\mathcal{S}=\mathrm{S} 5$. Then we can use it as a subroutine in the following algorithm.

Theorem 4.10 Given a modal logic $\mathcal{S}$ among S5, S4F, KD45, SW5, a theory $I$ and a partition $(\Phi, \Psi)$ of $M A(I)$, deciding whether $T_{I}(\Phi, \Psi)$ is not an $\mathcal{S}_{G}$-expansion for $I$ is a problem in $\Sigma_{2}^{p}$.

Proof We exhibit the following nondeterministic algorithm:

Algorithm Not-Ground $(\mathcal{S}, I,(\Phi, \Psi))$

Input: modal logic $\mathcal{S} \in\{\mathrm{S} 5, \mathrm{~S} 4 \mathrm{~F}, \mathrm{KD} 45, \mathrm{SW} 5\}$, theory $I$, partition $(\Phi, \Psi)$ of $M A(I)$;

Output: true if $T_{I}(\Phi, \Psi)$ is not an $\mathcal{S}_{G}$-expansion for $I$, false otherwise;

(1) if $(\Phi, \Psi)$ is not introspection-consistent with $I$ then return true;

(2) if $\operatorname{EXPANSION}(\mathcal{S}, I,(\Phi, \Psi))$ returns false then return true;

(3) if there exists partition $\left(\Phi^{\prime}, \Psi^{\prime}\right) \neq(\Phi, \Psi)$ such that:

(3.1) $\left(\Phi^{\prime}, \Psi^{\prime}\right)$ is introspection-consistent with $I$ and

(3.2) $T_{I}\left(\Phi^{\prime}, \Psi^{\prime}\right) \cap \mathcal{L} \subset T_{I}(\Phi, \Psi) \cap \mathcal{L}$ then return true;

(4) return false

Correctness of the algorithm follows from the theorems of this section and from Proposition 3.9. Regarding complexity, Steps 1 and 2 can be performed with a polynomial number of calls to an NP-oracle. Step 3 can be done by nondeterministically choosing $\left(\Phi^{\prime}, \Psi^{\prime}\right)$, and then verifying Conditions 3.1 and 3.2 again with a polynomial number of calls to an NPoracle. In particular, Condition 3.2 is verified by extracting the polynomial-size formulae representing the objective part of $T_{I}\left(\Phi^{\prime}, \Psi^{\prime}\right)$ and $T_{I}(\Phi, \Psi)$, respectively, then using propositional entailment.

The above result implies that groundedness can be decided with a $\Sigma_{2}^{p}$-oracle. We use this fact for establishing the upper bound on the complexity of reasoning in $\mathrm{S}_{G}, \mathrm{~S}_{\mathrm{F}} \mathrm{F}_{G}, \mathrm{KD} 45_{G}$ or $\mathrm{SW} 5_{G}$.

Theorem 4.11 Let $\mathcal{S}$ be one of the modal logics S5, S4F, KD45 or SW5. Entailment in $\mathcal{S}_{G}$ is in $\Pi_{3}^{p}$.

Proof We exhibit the following nondeterministic algorithm, which checks non-entailment: Algorithm Not-Entails $(\mathcal{S}, I, \varphi)$

Input: modal logic $\mathcal{S} \in\{\mathrm{S} 5, \mathrm{KD} 45, \mathrm{~S} 4 \mathrm{~F}, \mathrm{SW} 5\}$, theory $I$, formula $\varphi$;

Output: true if $I \not \forall_{\mathcal{S}_{G}} \varphi$, false otherwise.

(1) compute $M A(I)$;

(2) if there exists partition $(\Phi, \Psi)$ of $M A(I)$ such that

(2.1) Not-Ground $(\mathcal{S}, I,(\Phi, \Psi))$ returns false and

(2.2) $\neg \varphi \in T_{I}(\Phi, \Psi)$ then return true;

(3) return false 
Correctness of the algorithm immediately follows from the definition of entailment and the correctness of the previous algorithm Not-Ground. Step 1 is performed in polynomial time, and Step 2 can be accomplished by nondeterministically choosing a partition of $M A(I)$, and verifying that (2.1) it identifies an $\mathcal{S}_{G}$-expansion of $I$ with a $\Sigma_{2}^{p}$-oracle, and (2.2) $\neg \varphi$ can be deduced in $T_{I}(\Phi, \Psi)$ with a polynomial number of calls to an NP-oracle [20, Theorem 13.10]. Therefore, non-entailment is a problem in $\Sigma_{3}^{p}$, hence entailment is in $\Pi_{3}^{p}$.

Corollary 4.12 Let $\mathcal{S}$ be one of the modal logics S5, S4F, KD45 or SW5. Entailment in $\mathcal{S}_{G}$ is $\Pi_{3}^{p}$-complete.

\section{Representing knowledge}

In this section we discuss merits and drawbacks of ground logics as knowledge representation formalisms, through a comparison with McDermott and Doyle's logics. In particular, we address some features of these logics arising from both semantical and computational considerations, consider the representation of defaults and the use of explicit definitions.

\subsection{Semantics}

In Section 3 we have discussed the differences between the semantical definition of ground logics and McDermott and Doyle's logics. The criterion for choosing the preferred model is more selective in the case of ground logics since more monotonic models of the theory can be compared. Hence, every $\mathcal{S}_{G}$-expansion for $I$ is also an $\mathcal{S}_{M D D}$-expansion for $I$, while the converse in general does not hold.

A number of properties of ground logics, that we briefly outline in the following, can be derived from semantical considerations:

- none of the ground logics collapses into a monotonic logic (in particular for $\mathcal{S}=$ S5 the logic defined in [11] is obtained, as stated in [29]);

- $\mathrm{S}_{G}$ shows monotonicity with respect to objective formulae, in the sense that for each $I, \varphi \in \mathcal{L}_{K}$ and for each $\psi \in \mathcal{L}$, if $I \models \mathrm{S}_{G} \psi$ then $I \cup\{\varphi\} \models \mathrm{S}_{G} \psi$. Therefore, when adding new information in $\mathrm{S} 5_{G}$, only modal formulae can be lost, no longer being derivable in the resulting theory (see Example 2.3);

- the family $\mathcal{S}_{G}$ shows more variety than the family $\mathcal{S}_{M D D}$, in the sense that ranges of logics, that is, classes of modal logics with the same nonmonotonic counterpart (see [20, par.11.2]), are more common (and larger) in MDD logics than in ground logics. In other words, two ground logics hardly collapse in the same nonmonotonic logic.

\subsection{Computation}

In Section 4 we have shown that complexity of deduction in the family of ground logics is $\Pi_{3}^{p}$-hard. Hence, from the computational point of view, reasoning in a ground logic $\mathcal{S}_{G}$ is in general harder than reasoning in the corresponding $\mathcal{S}_{M D D}$ logic (unless $\Pi_{2}^{p}=\Pi_{3}^{p}=P H$ ), since entailment in $\mathrm{S}_{4} \mathrm{~F}_{M D D}, \mathrm{KD}_{M D} 5_{M D}$ and $\mathrm{SW} 5_{M D D}$ is $\Pi_{2}^{p}$-complete $([8,26,20])$. This increase in the complexity of reasoning is explained by the extra work which is necessary to make a further selection among $\mathcal{S}_{M D D}$-expansions so to restrict only to the ground ones. 
However, this source of complexity can be easily avoided when unnecessary. For example, it is well known that cautious reasoning in default logic is a $\Pi_{2}^{p}$-complete problem [8]. Hence, the translation of a default theory into a ground logic (see next subsection) seems to be disadvantageous, since it introduces an additional degree of complexity. On the other hand, the Not-Entails algorithm for ground logics presented above can be easily modified to make it work in $\Sigma_{2}^{p}$ in the 'easy' cases, i.e. for theories where $\mathcal{S}_{M D D}$-expansions and $\mathcal{S}_{G^{-}}$ expansions coincide. In fact, in such cases the groundedness check for the partition which identifies an $\mathcal{S}_{M D D}$-expansion (Step 3 of the algorithm Not-Ground) can be skipped, thus turning Not-Ground to a $P^{N P}$-algorithm, and Not-Entails to a $\Sigma_{2}^{p}$-algorithm. Since for theories obtained through a translation of a default theory we apriori know that $\mathcal{S}_{G}$-expansions and $\mathcal{S}_{M D D}$-expansions for such theories coincide, reasoning in a ground logic setting does not introduce additional complexity.

Moreover, there are other interesting cases in which the reasoning task is easier than in the general case. As pointed out in [11], entailment in $\mathrm{S} 5_{G}$ is in $P^{N P}$, if the theory admits only one $\mathrm{S}_{G}$-expansion. Such theories are called honest. The problem of deciding whether a theory $I$ is honest is in $P^{N P}$ as well. In particular, in [9] it is shown that deciding whether $I \models{ }_{5_{G}} \varphi$ when $I$ is the empty set is a $P^{N P}[O(\log n)]$-complete problem.

Finally, in [2] a first-order version of $\mathrm{S}_{G}$ is used to formalize some non-first-order features of frame systems, in particular procedural rules and a form of closed-world reasoning. It is shown that such restricted modal extension of a subset of first-order logic does not affect computational complexity of reasoning, which is PSPACE-complete both in the first-order and in the extended modal framework.

\subsection{Defaults}

In this section we discuss whether defaults are representable as epistemic sentences in ground logics. We show that logic $\mathrm{S} 5_{G}$ does not admit any modular translation for default theories, while such a translation is possible in the case of ground logics built from modal systems different from S5.

We first briefly recall default logic [27]. A propositional default theory is a pair $(D, W)$, such that $D$ is a set of defaults, i.e. inference rules of the form

$$
\frac{\alpha: M \beta_{1}, \ldots, M \beta_{n}}{\gamma}
$$

where $\alpha, \beta_{i}, \gamma \in \mathcal{L}, W$ is a theory in $\mathcal{L}$ and $M \beta$ is interpreted as: "it is consistent to assume $\beta$ ". A justification-free default is a default where the justification part is empty, i.e. of the form

$$
\frac{\alpha:}{\gamma}
$$

A prerequisite-free default is a default of the form

$$
\frac{: M \beta_{1} \ldots M \beta_{n}}{\gamma}
$$

We are interested in the translation of a default theory into a modal theory. Therefore we give the following definitions (taken from [10]) and recall some properties.

Definition 5.1 A faithful translation from default logic to a ground nonmonotonic logic $\mathcal{S}_{G}$ is a mapping $\operatorname{tr}$ which transforms each default theory $(D, W)$ into a modal theory $\operatorname{tr}(D, W)$ 
such that the objective (i.e. non-modal) parts of the $\mathcal{S}_{G}$-expansions of $\operatorname{tr}(D, W)$ are exactly the default extensions of $(D, W)$.

However, not every translation that is faithful is useful in practice. In particular, we would like to be able to turn each default into a modal sentence, independently of other defaults and of the theory. Such translations are called modular.

Definition 5.2 A translation $t r$ from default logic to a modal nonmonotonic logic $\mathcal{S}$ is $\bmod$ ular iff for each default set $D$ and each $W \subseteq \mathcal{L}$ it holds that $\operatorname{tr}(D, W)=\operatorname{tr}(D, \emptyset) \cup W$.

We shall use the modular translation emb introduced in [34].

$$
\begin{gathered}
e m b(d)=K \alpha \wedge K \neg K \neg \beta_{1} \wedge \ldots \wedge K \neg K \neg \beta_{n} \supset \gamma \\
e m b(D, W)=W \cup\{e m b(d) \mid d \in D\}
\end{gathered}
$$

where $d$ is a default.

Proposition 5.3 [20, 12.1] Let $\mathcal{S}$ be a modal logic such that $\mathrm{K} \subseteq \mathcal{S} \subseteq \mathrm{S} 4 \mathrm{~F}$. Let $S \subseteq \mathcal{L}$ be consistent and closed under propositional consequence. Then $S$ is an extension for a default theory $(D, W)$ iff $S T(S)$ is an $\mathcal{S}_{M D D}$-expansion for $\operatorname{emb}(D, W)$.

We now show some properties of ground logics with respect to the representation of defaults.

A first interesting result concerns the existence of modular translations for justificationfree defaults. In particular, we have that $\operatorname{emb}(D, W)$ provides the desired result for any ground nonmonotonic logic. Notice that the same translation is used in [2] to formalize procedural rules of frame systems within a concept language augmented by a modal operator interpreted in $\mathrm{S}_{G}$.

Theorem 5.4 There exists a faithful modular translation from justification-free default theories to any ground nonmonotonic logic.

Proof Let $(D, W)$ be a default theory such that $D$ is a collection of justification-free defaults. Then $(D, W)$ has exactly one default extension $S$. The theory $\operatorname{emb}(D, W)$ has $S T(S)$ as its only $\mathrm{K}_{G}$-expansion (see Theorem 5.6 below). Moreover, it can be shown that every S5model $\mathcal{M}$ for $\operatorname{emb}(D, W)$ is such that $S \subseteq T h(\mathcal{M}) \cap \mathcal{L}$. This implies that $S T(S)$ is the only $\mathrm{S}_{G}$-expansion for $\operatorname{emb}(D, W)$. Thus, for every logic $\mathcal{S}$ such that $\mathrm{K} \subseteq \mathcal{S} \subseteq \mathrm{S} 5$, theory $\operatorname{emb}(D, W)$ admits exactly one $\mathcal{S}_{G}$-expansion $S T(S)$. Therefore for such logics emb is a faithful translation for justification-free defaults.

It is interesting to compare this result with an analogous property of McDermott and Doyle's logics, which states that every logic $\mathcal{S}_{M D D}$ contained in 55 admits a faithful modular translation for prerequisite-free default theories [10]. On the other hand, there exist logics $\mathcal{S}_{M D D}$ for which a faithful modular translation from justification-free default theories to $\mathcal{S}_{M D D}$ is impossible. Therefore, McDermott and Doyle's logics and ground logics show a complementary behaviour in that the former can always represent prerequisite-free defaults, while the latter can always represent justification-free defaults. We provide futher evidence to this phenomenon by first showing that defaults cannot be represented in the logic $\mathrm{S} 5_{G}$, then showing that prerequisite-free defaults can be represented in $\mathrm{KD} 45_{G}$. 
Theorem 5.5 There exists no faithful modular translation from default logic to $\mathrm{S} 5_{G}$.

Proof Consider the default theory $\left(D, W_{0}\right)$ such that

$$
\begin{gathered}
D=\left\{\frac{: p}{p}\right\} \\
W_{0}=\emptyset
\end{gathered}
$$

Suppose $t r$ is a faithful modular translation from default logic to $\mathrm{S} 5_{G}$. Faithfulness of $t r$ implies that $\operatorname{tr}\left(D, W_{0}\right)$ has only one $\mathrm{S}_{G}$-expansion $T=S T(\{p\})$. Therefore, in every S5model $\mathcal{M}$ for $\operatorname{tr}\left(D, W_{0}\right)$ it holds that $\mathcal{M} \models p$. Now, given $W_{1}=\{\neg p\}$, by the hypothesis of modularity of $\operatorname{tr}$ it follows that $\operatorname{tr}\left(D, W_{1}\right)=\operatorname{tr}\left(D, W_{0}\right) \cup W_{1}$. Consequently, $\operatorname{tr}\left(D, W_{1}\right)$ is an S5-inconsistent theory, and hence it has no $\mathrm{S} 5_{G}$-expansions, while on the other hand the default theory $\left(D, W_{1}\right)$ has the default extension $C n(\{\neg p\})$, thus contradicting the hypothesis of faithfulness of $t r$.

The impossibility of a faithful modular translation from default theories to $\mathrm{S} 5_{G}$ originates from the monotonicity of this particular logic with respect to objective formulae (see Section 5.1): in $S 5_{G}$ only modal formulae can change their validity when new information is added. Since no other ground logic shares this characteristic with $\mathrm{S}_{G}$, this negative behaviour seems to be restricted to the logic $\mathrm{S} 5_{G}$ only. In fact, for a wide class of ground logics we obtain the following positive result ${ }^{2}$ (analogous to that obtained for McDermott and Doyle's logics in [34]).

Theorem 5.6 Given a modal logic $\mathcal{S}$ such that $\mathrm{K} \subseteq \mathcal{S} \subseteq \mathrm{S} 4 \mathrm{~F}$, there exists a faithful modular translation from default logic to the ground nonmonotonic logic $\mathcal{S}_{G}$.

Proof First, we show that if $S$ is a default extension for the default theory $(D, W)$, then $S T(S)$ is an $\mathcal{S}_{G}$-expansion for $\operatorname{emb}(D, W)$ for any modal logic $\mathcal{S}$ such that $\mathrm{K} \subseteq \mathcal{S} \subseteq \mathrm{S} 5$. In fact, it can be easily shown that

1. $S \subseteq C n_{\mathrm{K}}(\operatorname{emb}(D, W) \cup\{\neg K \varphi \mid \varphi \in \mathcal{L} \backslash S\})$;

2. $\operatorname{emb}(D, W) \subseteq S T(S)$.

And since $S T(S)$ is stable and consistent, it follows from Proposition 3.5 that $S T(S)$ is a $\mathrm{K}_{G}$-expansion for $\operatorname{emb}(D, W)$.

Then, we prove that if $S T(S)$ is an $\mathrm{S}_{4} \mathrm{~F}_{G}$-expansion for $\operatorname{emb}(D, W)$, then $S$ is a default extension for $(D, W)$. This is obtained by exploiting a correspondence between minimal expansions in McDermott and Doyle's logics and ground expansions. In fact, from Proposition 5.3 it follows that $e m b$ is a faithful translation for S4F $M D D$. Moreover, logic S4F satisfies the terminal cluster property, therefore by Theorem 3.9 S4F $\mathrm{F}_{G}$-expansions exactly correspond to minimal $\mathrm{S}_{4} \mathrm{~F}_{M D D}$-expansions. Now, the default extensions for $(D, W)$ correspond to the objective parts of the $\mathrm{S}_{4} \mathrm{~F}_{M D D}$-expansions for $\operatorname{emb}(D, W)$, and since every default extension is minimal (i.e. it cannot be the case that $S \subset S^{\prime}$ for any pair of default extensions $S, S^{\prime}$ ), it follows that the $\mathrm{S}_{4} \mathrm{~F}_{G}$-expansions for $\operatorname{emb}(D, W)$ are exactly the $\mathrm{S}_{4} \mathrm{~F}_{M D D}$-expansions for $e m b(D, W)$. And since $e m b$ is a faithful translation for $\mathrm{S}_{4} \mathrm{~F}_{M D D}$, this proves that if $S T(S)$ is a $44 \mathrm{~F}_{G}$-expansion for $\operatorname{emb}(D, W)$, then $S$ is a default extension for $(D, W)$.

\footnotetext{
${ }^{2}$ This theorem was independently proved in [35].
} 
Now, from Proposition 3.6, it follows that for every modal logic $\mathcal{S}$ such that $\mathrm{K} \subseteq \mathcal{S} \subseteq$ $\mathrm{S} 4 \mathrm{~F}$, if $S T(S)$ is an $\mathcal{S}_{G}$-expansion for $\operatorname{emb}(D, W)$, then it is an $\mathrm{S} \mathrm{F}_{G}$-expansion for $\operatorname{emb}(D, W)$, which implies that $S$ is a default extension of $(D, W)$.

The two above results imply that, for each modal logic $\mathcal{S}$ such that $\mathrm{K} \subseteq \mathcal{S} \subseteq \mathrm{S} 4 \mathrm{~F}$, emb is a faithful translation for $\mathcal{S}$; since $e m b$ is modular, this concludes the proof.

The next result concerns a class of logics comprising $\mathrm{KD} 45_{G}$, i.e. Konolige's moderately grounded version of autoepistemic logic [14].

Theorem 5.7 For every logic $\mathcal{S}$ such that $\mathrm{K} \subseteq \mathcal{S} \subseteq \mathrm{KD} 45$, there exists a faithful modular translation from prerequisite-free default theories to ground nonmonotonic logic $\mathcal{S}_{G}$.

Proof Theorem 2.2 in [10], states that the following modular translation $\tau$ :

$$
\begin{gathered}
\tau\left(\frac{: M \beta_{1} \ldots M \beta_{n}}{\gamma}\right)=\neg K \neg \beta_{1} \wedge \ldots \wedge \neg K \neg \beta_{n} \supset \gamma \\
\tau(D, W)=W \cup\{\tau(d) \mid d \in D\}
\end{gathered}
$$

is a faithful translation from prerequisite-free default theories to $\mathrm{KD} 45_{M D D}$. Besides, logic KD45 satisfies the terminal cluster property, therefore by Theorem $3.9 \mathrm{KD} 45_{G}$-expansions exactly correspond to minimal KD45 $M D D$-expansions. Following the proof of previous theorem, in an analogous way we come to the conclusion that the $\mathrm{KD} 45_{G}$-expansions for $\tau(D, W)$ are exactly the $\mathrm{KD} 45_{M D D}$-expansions for $\tau(D, W)$. Moreover, following the proof given for the translation $e m b$, it can easily be shown that translation $\tau$ is such that, if $S$ is a default extension of the default theory $(D, W)$, then $S T(S)$ is a $\mathrm{K}_{G}$-expansion of theory $\tau(D, W)$. This proves that $\tau$ is a faithful translation of prerequisite-free default theories for every logic $\mathcal{S}$ such that $\mathrm{K} \subseteq \mathcal{S} \subseteq \mathrm{KD} 45$.

\subsection{Definitions}

Finally, we addess an issue arising with definitions and outlined by the following example [31]. Let $I=\emptyset$. Clearly, according to minimal knowledge, $\neg K p$ can be concluded for every propositional letter $p$. Now, if the sentence $q \equiv K p$ is added to $I, \neg K p$ can no longer be concluded, which is undesirable, because $q \equiv K p$ is regarded as the explicit definition of $q$, which should leave everything not concerning $q$ unchanged.

As admitted in [31] the example is "controversial", and it requires a better understanding of the intuition of this kind of formulae. In fact, sentences of the form $q \equiv K p$ can be rephrased as $(K p \supset q) \wedge(\neg K p \supset \neg q)$, which can be seen as the conjunction of the translation of two defaults: a justification-free and a prerequisite-free one. Therefore, it seems that one can hardly consider the combination of the two as a definition in the classical sense. Sentences of the form $q \equiv K p$ should thus be treated as a special class of epistemic sentences whose role still appears unclear. In fact, we believe that the unpleasant behaviour of ground logics in the example above is not to be interpreted as a fault of the logic, but rather as a loss of definitions as double implications. In practical environments this would simply mean that definitions in the classical fashion are appropriate for objective sentences, while the role of implications involving modal formulae is currently understood only for particular sentences, such as those arising from the translation of defaults. 


\section{Conclusions}

Ground logics are defined following the intuition that the nonmonotonic assumptions made by an agent should be done by minimizing the set of objective sentences known by the agent itself. We have discussed the features of ground logics with regard to their use in knowledge representation. In particular, we have investigated their semantics and both their computational and epistemological properties.

The results of this work can be given a twofold reading: as a complete semantical and computational characterization of ground logics; as a generalization of the semantical approach to nonmonotonic modal logics based on the minimization of the knowledge of the agent.

The main conclusion of the work is that ground logics are more powerful than McDermott and Doyle's logics. In fact, they allow the agent to derive statements about its lack of knowledge, or ignorance, which cannot be obtained in McDermott and Doyle's logics. This additional expressivity is further demonstrated by a more complex reasoning required in the general case. Moreover, ground logics can be regarded as an incremental refinement of McDermott and Doyle's logics, since they simply discard some of their models, while showing a similar behaviour in many of their uses, such as for example the representation of defaults.

Finally, the ground logic for S5, which has no counterpart in the McDermott and Doyle's family, is interesting in its own. Such a logic, based on the idea of minimal knowledge [11], has both an intuitive interpretation and has been successfully applied in the formalization of several features of knowledge representation systems based on description logics [2].

\section{Acknowledgements}

We thank Marco Cadoli and Thomas Eiter for their careful reading of the paper, their comments and suggestions for the improvement of the presentation. We also thank two anonymous referees for their helpful comments. This research has been funded by MURST, "Tecniche di Ragionamento Non Monotono".

\section{References}

[1] A. Bochman. On bimodal nonmonotonic modal logics and their unimodal and nonmodal equivalents. In Proceedings of the Fourteenth International Joint Conference on Artificial Intelligence (IJCAI-95), pages 1518-1524, 1995.

[2] F. M. Donini, M. Lenzerini, D. Nardi, W. Nutt, and A. Schaerf. Adding epistemic operators to concept languages. In Proceedings of the Third International Conference on the Principles of Knowledge Representation and Reasoning (KR-92), pages 342-353. Morgan Kaufmann, Los Altos, 1992.

[3] F. M. Donini, F. Massacci, D. Nardi, and R. Rosati. A uniform tableau method for nonmonotonic modal logics. In J.J. Alferes, L.M. Pereira and E. Orlowska, editors, Logics in Artificial Intelligence - Proceedings of JELIA'96, number 1126 in Lecture Notes In Artificial Intelligence, pages 87-103. Springer-Verlag, 1996.

[4] F. M. Donini, D. Nardi, and R. Rosati. Ground nonmonotonic modal logics for knowledge representation. In M. De Glas and Z. Pawlak, editors, Proceedings of the Second World Conference on the Fundamentals of Artificial Intelligence (WOCFAI-95), pages 133-144. Angkor, Paris, 1995.

[5] T. Eiter and G. Gottlob. Reasoning with parsimonious and moderately grounded expansions. Fundamenta Informaticae, 17(1,2):31-54, 1992.

[6] J. Engelfriet. Minimal temporal epistemic logic. Technical report IR388, Vrjie Universiteit Amsterdam, 1995.

[7] M. L. Ginsberg, editor. Readings in Nonmonotonic Reasoning. Morgan Kaufmann, Los Altos, 1987.

[8] G. Gottlob. Complexity results for nonmonotonic logics. Journal of Logic and Computation, 2:397-425, 1992.

[9] G. Gottlob. NP trees and Carnap's modal logic. Journal of the ACM, 42(2):421-457, 1995.

[10] G. Gottlob. Translating default logic into standard autoepistemic logic. Journal of the ACM, 42(4):711-740, 1995.

[11] J. Y. Halpern and Y. Moses. Towards a theory of knowledge and ignorance: Preliminary report. Technical Report CD-TR 92/34, IBM, 1985.

[12] D. S. Johnson. A catalog of complexity classes. In J. van Leeuwen, editor, Handbook of Theoretical Computer Science, volume A, chapter 2. Elsevier Science Publishers B. V. (North Holland), 1990. 
[13] M. Kaminski. Embedding a default system into nonmonotonic modal logics. Fundamenta Informaticae, 14:345-354, 1991.

[14] K. Konolige. On the relation between default and autoepistemic logic. Artificial Intelligence Journal, 35:343-382, 1988.

[15] V. Lifschitz. Nonmonotonic databases and epistemic queries. In Proceedings of the Twelfth International Joint Conference on Artificial Intelligence (IJCAI-91), pages 381-386, Sydney, 1991.

[16] V. Lifschitz. Minimal belief and negation as failure. Artificial Intelligence Journal, 70:53-72, 1994.

[17] F. Lin and Y. Shoham. Epistemic semantics for fixed-point non-monotonic logics. Artificial Intelligence Journal, 57:271-289, 1992.

[18] W. Marek, G. Shvarts, and M. Truszczyński. Modal nonmonotonic logics: ranges, characterization, computation. In Proceedings of the Second International Conference on the Principles of Knowledge Representation and Reasoning (KR-91), pages 395-404. Morgan Kaufmann, Los Altos, 1991.

[19] W. Marek and M. Truszczyński. Autoepistemic logic. Journal of the ACM, 38(3):588-619, 1991

[20] W. Marek and M. Truszczyński. Nonmonotonic Logics - Context-Dependent Reasoning. Springer-Verlag, 1993.

[21] D. McDermott. Non-monotonic logic II: Non-monotonic modal theories. Journal of the ACM, 29:33-57, 1982.

[22] D. McDermott and J. Doyle. Non-monotonic logic I. Artificial Intelligence Journal, 13:41-72, 1980.

[23] J.-J. Ch. Meyer and W. van der Hoek. A Default Logic Based on Epistemic States. Fundamenta Informaticae, 23, 1 (1995) pp. $33-65$.

[24] R. C. Moore. Semantical considerations on nonmonotonic logic. Artificial Intelligence Journal, 25:75-94, 1985.

[25] D. Nardi and R. Rosati. A preference semantics for ground nonmonotonic modal logics. In Proceedings of the 7th Portuguese Conference on Artificial Intelligence (EPIA-95), number 990 in Lecture Notes In Artificial Intelligence, pages 225-236. Springer-Verlag, 1995.

[26] I. Niemelä. Decision procedure for autoepistemic logic. In Proceedings of the Ninth International Conference on Automated Deduction (CADE-88), volume 310 of Lecture Notes in Computer Science, pages 675-684. Springer-Verlag, 1988.

[27] R. Reiter. A logic for default reasoning. Artificial Intelligence Journal, 13:81-132, 1980. Republished in [7].

[28] G. Schwarz. Autoepistemic modal logics. In Proceedings of the Third Conference on Theoretical Aspects of Reasoning about Knowledge (TARK-90), pages 97-109, 1990.

[29] G. Schwarz. Bounding introspection in nonmonotonic logics. In Proceedings of the Third International Conference on the Principles of Knowledge Representation and Reasoning (KR-92), pages 581-590. Morgan Kaufmann, Los Altos, 1992.

[30] G. Schwarz. Minimal model semantics for nonmonotonic modal logics. In Proceedings of the Seventh International Conference of Logic in Computer Science (LICS-92), pages 34-43. IEEE Computer Society Press, 1992.

[31] G. Schwarz and M. Truszczyński. Minimal knowledge problem: a new approach. Artificial Intelligence Journal, 67:113-141, 1994.

[32] Y. Shoham. Nonmonotonic logics: Meaning and utility. In Proceedings of the Tenth International Joint Conference on Artificial Intelligence (IJCAI-87), pages 388-392, 1987.

[33] M. Tiomkin and M. Kaminski. Nonmonotonic default modal logics. In Proceedings of the Third Conference on Theoretical Aspects of Reasoning about Knowledge (TARK-90), pages 73-84, 1990.

[34] M. Truszczyński. Modal interpretations of default logic. In Proceedings of the Twelfth International Joint Conference on Artificial Intelligence (IJCAI-91), pages 393-398, 1991.

[35] M. Truszczyński. Embedding default logic into modal nonmonotonic logics. In Proceedings of LPNMR-91, pages 151-165, MIT Press, 1991.

[36] M. Truszczyński. Modal nonmonotonic logic with restricted application of the negation as failure to prove rule. Fundamenta Informaticae, 14:355-366, 1991.

\section{Received 7 November 1995}

